\title{
Resilient habitability of nearby exoplanet systems
}

\author{
Giorgi Kokaia $^{1 \star}$, Melvyn B. Davies ${ }^{1}$, Alexander J. Mustill ${ }^{1}$ \\ ${ }^{1}$ Lund Observatory, Department of Astronomy and Theoretical Physics, Lund University, Box 43, SE-221 00 Lund, Sweden
}

4 December 2019

\begin{abstract}
We investigate the possibility of finding Earth-like planets in the habitable zone of 34 nearby FGK-dwarfs, each known to host one giant planet exterior to their habitable zone detected by RV. First we simulate the dynamics of the planetary systems in their present day configurations and determine the fraction of stable planetary orbits within their habitable zones. Then, we postulate that the eccentricity of the giant planet is a result of an instability in their past during which one or more other planets were ejected from the system. We simulate these scenarios and investigate whether planets orbiting in the habitable zone survive the instability. Explicitly we determine the fraction of test particles, originally found in the habitable zone, which remain in the habitable zone today. We label this fraction the resilient habitability of a system. We find that for most systems the probability of planets existing [or surviving] on stable orbits in the habitable zone becomes significantly smaller when we include a phase of instability in their history. We present a list of candidate systems with high resilient habitability for future observations. These are: HD 95872, HD 154345, HD 102843, HD 25015, GJ 328, HD 6718 and HD 150706. The known planets in the last two systems have large observational uncertainties on their eccentricities, which propagate into large uncertainties on their resilient habitability. Further observational constraints of these two eccentricities will allow us to better constrain the survivability of Earth-like planets in these systems.
\end{abstract}

Key words: planets and satellites: dynamical evolution and stability - planets and satellites: general - planets and satellites: terrestrial planets - planetary systems

\section{INTRODUCTION}

One of the key goals for exoplanet science is to observe an Earthlike planet located in the habitable zone of a Sun-like star, where the habitable zone is defined as the spherical shell at a distance that permits liquid water to exist on the planet's surface.

Detecting Earth-mass (i.e. low-mass) planets has been technologically limited, however new instruments such as ESPRESSO (currently operational with the sensitivity slowly increasing Pepe et al. 2010; Leite et al. 2018), space missions like PLATO (Rauer et al. 2014) and future large telescopes such as E-ELT and TMT (Gilmozzi \& Spyromilio 2007; Skidmore et al. 2015) offer the prospect for the discovery of Earth-like planets in the near future.

Discovering Earth-like planets will, however, be difficult even with the next generation of instruments. One must focus attention on a relatively small number of stars (or planetary systems). A natural question is then: which systems do we select for further detailed study? In this paper we will consider local stars possessing one observed (massive) planet located beyond the habitable zone. Through numerical modelling of their dynamical past, we select the subset having the best prospects for possessing a habitable low-

* Contact e-mail: giorgi@astro.lu.se mass planet. Considering systems containing a Jupiter-mass planet further out makes sense for a number of reasons:

(i) Recent work has shown that the presence of Jupiter was instrumental in shaping the inner parts of the Solar System by clearing out or preventing the delivery of a large fraction of the solids in the early inner disc. This might be a requirement for systems such as the Solar System to form (Batygin \& Laughlin 2015; Childs et al. 2019; Lambrechts et al. 2019), rather than the systems commonly found by Kepler which consist of super-Earths and mini-Neptunes on tightly packed orbits within 0.5 au (Mullally et al. 2015).

(ii) Until relatively recently there was commonly held belief that Jupiter protected the Earth from impacts (see e.g. Ward et al. 2000). This belief has come under scrutiny as the effect of Jupiter on impact rates has been looked at more closely through numerical simulations (Laakso et al. 2006). Jupiter actually appears to cause a slight enhancement in impacts from bodies originating in the asteroid and Kuiper belt (Horner \& Jones 2008, 2009) whilst reducing the impact rate of long-period comets (Horner et al. 2010). Regardless, giant planets appear to have been instrumental in delivering water and other volatiles to the inner parts of the system, whether it happened after formation (Chyba 1990; Morbidelli et al. 2000; Abramov \& Mojzsis 2009; Carter-Bond et al. 2014; Grazier 2016) or during formation (Raymond et al. 2006; Raymond \& Izidoro 2017; O’Brien et al. 2018). 
(iii) Systems with gas giants are very likely to also host lowmass planets. Recent studies have shown that $\sim 40 \%$ of systems that have low-mass planets (in this case "low-mass" implies $\lesssim 10 \mathrm{M}_{\oplus}$ ) also host an external gas giant (Zhu \& Wu 2018; Bryan et al. 2019). Further, Zhu \& Wu (2018) invert this occurrence rate using overall occurrence rates for each type of planet and conclude that $\sim 90 \%$ of gas giant systems should have interior, lower-mass planets. The correlations found are not strictly for the Earth-like planets that we consider in this work. However, one could argue that if there were data to consider these planets then the correlation might be even stronger, given the fact that a gas giant might be required to even be able to have Earth-like planets. For comparison, Burke et al. (2015) determined the occurrence rate for Earth-like planets around GK-dwarfs given by extrapolation from Kepler systems to be $\sim 6 \pm 3 \%$ for planets with radii between 0.75 and 1.75 Earth radii and 300-700 day orbits. By instead considering Kepler non-detections, Hsu et al. (2019) determined an upper bound (84 ${ }^{\text {th }}$ percentile) of $27 \%$ on the occurrence rate of planets with radii between 0.75 and 1.5 Earth radii on periods of $237-500$ days. They caution however that the bound is likely overestimated by up to a factor of two due to contamination by false alarms.

(iv) The RV-signal strength from a planet is proportional to $M_{\mathrm{p}} \sin I_{\mathrm{p}}$ where $I_{\mathrm{p}}$ is the inclination of the orbital plane with respect to the plane of the sky, with the observer at $I_{\mathrm{p}}=90^{\circ}$. For nearby giant planets, Gaia will be able to constrain the orbital plane (Sozzetti et al. 2008; Perryman et al. 2014; Ranalli et al. 2018). Given that planetary systems seem to typically have low mutual inclinations (Lissauer et al. 2011; Johansen et al. 2012), we can further constrain which systems to observe by picking out those in which the giant planet has $I_{\mathrm{p}} \approx 90^{\circ}$. This gives a strong prior for the amplitude of the RV signal from the Earth-like planet being observed to be close to its maximum possible value.

However, a gas giant may destabilise certain orbits including those within the habitable zone of a planetary system (Jones et al. 2001; Menou \& Tabachnik 2003; Veras \& Armitage 2005; Matsumura et al. 2013; Agnew et al. 2017, 2018; Georgakarakos et al. 2018). In this paper we will not only re-examine the present day habitability of systems containing giant planets, but also determine the fraction of test particles remaining within habitable zones when one accounts for the past dynamical evolution of particular systems. We term this fraction the resilient habitability (Carrera et al. 2016) of these systems

The resilient habitability of a system is determined by assuming that the system initially consisted of multiple planets and that the eccentricity of the observed planet is the result of one or more other planets' being ejected when the system became unstable. The idea is that changes to the orbits and ejection of planets during the instability will do considerably more damage to Earth-like planets in the habitable zone than the system as seen today with its postinstability architecture. In summary, from our simulations we determine two types of habitability for each of the observed systems we study:

Present-day habitability: The fraction of test particles that remain in the habitable zone when the system is simulated in its present day configuration. We denote this as $f_{\text {hab,1P. }}$.

Resilient habitability: The fraction of test particles that remain in the habitable zone when the past dynamical evolution of the system is simulated. We denote this as $f_{\text {rhab }}$.

This paper is arranged as follows. In section 2 we discuss how scattering between two planets can lead to the ejection of one with the other becoming more bound and eccentric. We show how the eccentricity reached is a function of the mass ratio of the two planets. In section 3 we present the known planet-hosting systems we have selected to investigate, each of which has a single giant planet exterior to the habitable zone. Section 4 describes our calculations of both the present day and resilient habitability of these systems. The results of these calculations are presented in section 5. The implications of our results are discussed in section 6 . In section 7 we list the systems which in our view represent the best candidates for further searches for Earth-like planets. We summarise the paper in section 8 .

\section{GIANT PLANET SCATTERING}

We know from observations (Buchhave et al. 2018), and suspect from simulations (Thommes et al. 2008; Mordasini et al. 2009), that giant planets rarely form alone. Furthermore, it has been shown that giant planets form on circular or nearly circular orbits (Bitsch et al. 2013; Ragusa et al. 2018) which are later made more eccentric, whereas the observed exoplanet eccentricity distribution is consistent with most of them having been made eccentric through planetplanet scattering (Ford \& Rasio 2008; Jurić \& Tremaine 2008).

In this section we consider scattering between massive planets within two-planet systems. When two planets are placed on sufficiently close, low-eccentricity orbits, they will become unstable, leading to the two orbits crossing which then leads to a series of strong planet-planet scattering events, with one planet being ejected whilst one remains on a more bound, and eccentric, orbit. For a review on the dynamical evolution of planetary systems see Davies et al. (2014).

Here, we model two systems orbiting around a Solar-mass star: one containing two Jupiter-mass planets and one containing one Jupiter-mass planet and one Saturn-mass planet, in order to investigate how the eccentricity distribution of the planets left behind (once one planet is ejected) depends on the mass ratio of the two planets. We place one Jupiter at 5.2 au and place a second either Jupiter- or Saturn-mass planet in the range [5.2,7.6] au. This setup eventually leads to planet-planet scattering during which either of the Jupiter-mass planets can be ejected in the first case and the Saturn-mass planet is ejected in the second case. Figure 1 shows the semi-major axis and eccentricity of the remaining planet for the two systems. The distribution of eccentricities is clearly a function of the mass ratio of the two planets. In ejecting a second Jupiter, the remaining Jupiter-mass planet has to give up more energy and angular momentum, thus making it more bound and more eccentric than when the inner Jupiter ejects a Saturn-mass planet.

We will make use of the mass-ratio dependence of eccentricity when we consider the dynamical histories of our selected observed systems. For example, if the observed planet in a system has a eccentricity below about 0.3 , we can then expect the planet to have ejected (only) Saturn-mass planets in the past, whereas for larger eccentricities, the planet is more likely to have ejected one (or more) planets of equal mass.

\section{SYSTEM SELECTION}

We select systems from the exoplanet $\operatorname{archive}^{1}$ with a single gas giant $\left(0.3 \mathrm{M}_{\mathrm{J}}<M_{\mathrm{p}} \sin I_{\mathrm{p}}<8 \mathrm{M}_{\mathrm{J}}\right.$ where $\mathrm{M}_{\mathrm{J}}$ is the mass of

\footnotetext{
1 https://exoplanetarchive.ipac.caltech.edu/
} 


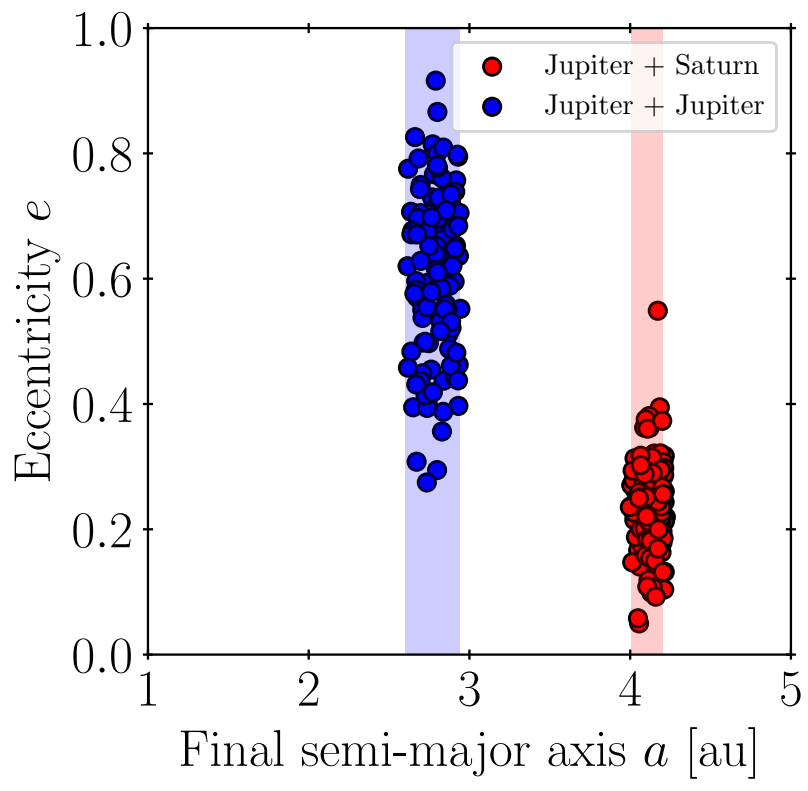

Figure 1. The figure shows the $a$ and $e$ of a surviving Jupiter-mass planet after having ejected a second planet in an unstable two-planet system. The shaded region shows the range of semi-major axes permitted based on a simple energy consideration. The two colours show two different mass combinations: Jupiter plus Jupiter, and Jupiter plus Saturn.

Table 1. Parameters used in equation 2 for determining the boundaries of the habitable zone as given by Kopparapu et al. (2014)

\begin{tabular}{lrr} 
Parameter & \multicolumn{1}{c}{$\begin{array}{c}\text { Inner edge } \\
\text { Runaway Greenhouse }\end{array}$} & \multicolumn{1}{c}{$\begin{array}{c}\text { Outer edge } \\
\text { Maximum Greenhouse }\end{array}$} \\
\hline$S_{\text {eff } \odot}$ & 1.107 & 0.356 \\
$a$ & $1.332 \times 10^{-4}$ & $6.171 \times 10^{-5}$ \\
$b$ & $1.58 \times 10^{-8}$ & $1.698 \times 10^{-9}$ \\
$c$ & $-8.308 \times 10^{-12}$ & $-3.198 \times 10^{-12}$ \\
$d$ & $-1.931 \times 10^{-15}$ & $-5.575 \times 10^{-16}$
\end{tabular}

Jupiter) orbiting an FGK-dwarf $\operatorname{star}\left(1.4 \mathrm{M}_{\odot} \gtrsim \mathrm{M}_{\star} \gtrsim 0.65 \mathrm{M}_{\odot}\right.$, $\mathrm{R}_{\star}<2 R_{\odot}$ ) beyond its habitable zone (HZ).

The habitable zone (HZ) is defined as a shell at a distance from a star with a given luminosity $(L)$ and effective temperature $\left(T_{\text {eff }}\right)$ in which a planet can have liquid water on its surface. The boundaries of the $\mathrm{HZ}$ for each system are determined using the formula derived by Kopparapu et al. (2014), shown below:

$d=\left(\frac{L / L_{\odot}}{S_{\mathrm{eff}}}\right)^{0.5} \mathrm{AU}$

where $L_{\odot}$ is the Solar luminosity and

$S_{\text {eff }}=S_{\text {eff } \odot}+a T_{\star}+b T_{\star}^{2}+c T_{\star}^{3}+d T_{\star}^{4}$

is a measure of the flux received by the planet and $T_{\star}=T_{\text {eff }}-T_{\odot}$ $\left(T_{\odot}=5780 K\right)$. The values for the parameters are given in table 1 . The inner edge of the $\mathrm{HZ}$ is set using the model parameters for runaway greenhouse habitability, the limit at which the temperature of the planet, under any realistic atmospheric conditions, inevitably becomes too high. The outer edge is set using the maximum greenhouse parameters, which is the limit at which an optimally constructed atmosphere to maximise the greenhouse effect can no longer prevent all the water from freezing.

To further limit the number of systems we simulate, we impose another criterion; the pericentre calculated using an eccentric-

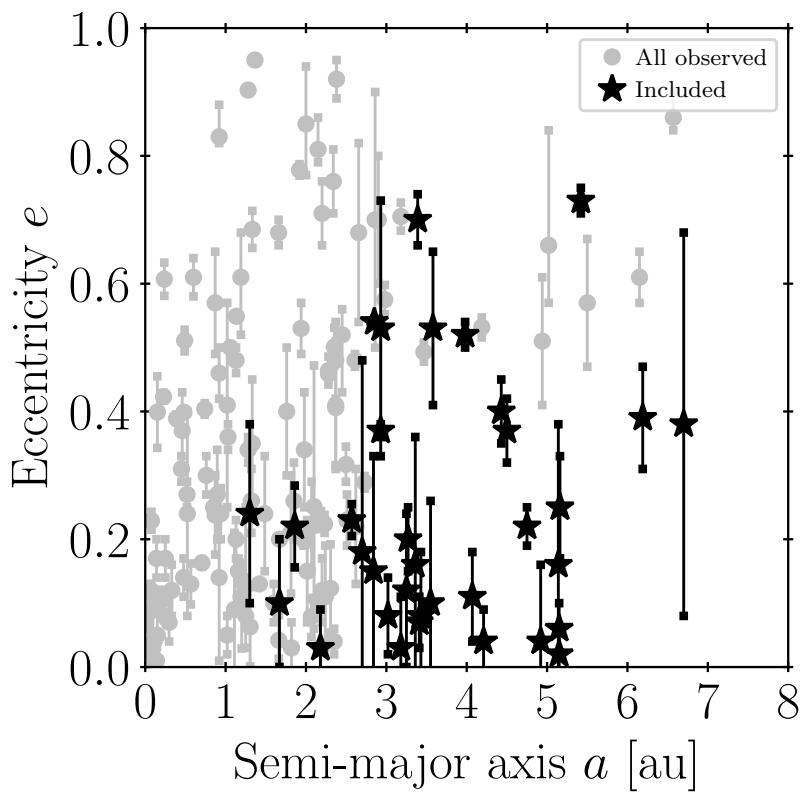

Figure 2. All of the known single-planet RV systems that meet our selection criteria for planet mass, and for stellar mass and radius. The black stars in the plot show the systems in table 2; the grey markers are systems which meet the selection criteria but where the planet has an orbit that enters, is inside or interior to the habitable zone.

ity one standard deviation lower than the mean observed value must lie exterior to the $\mathrm{HZ}$.

The criteria leave us with 34 systems as listed in table 2 . We include in this table the stellar parameters used to determine the HZ. They are plotted and highlighted in figure 2 along with every other known single planet RV system that matches our criterion for planetary mass and stellar type. Half of these systems can be found on the short-list of Agnew et al. $(2017,2018)$ who investigated the present day stability of orbits in the HZ. For the remaining half, some planets were considered by Agnew et al. $(2017,2018)$ to be so distant that the orbits of test particles in the $\mathrm{HZ}$ would be unaffected by the planet, and therefore were not investigated. We also include some high eccentricity systems with limited stability that did not make their cut and some systems discovered subsequent to their work.

\section{SETUP OF THE NUMERICAL EXPERIMENTS}

We perform all our $N$-body simulations using the Bulirsch-Stoer integrator of the MERCURY package (Chambers 1999), with an accuracy parameter of $10^{-12}$ resulting in a relative energy error of less than $10^{-5}$ for all runs.

\subsection{Present-day habitability}

As a first step we simulate all the planetary systems in their present day configuration to determine the present day habitability, i.e. the fraction of test particle orbits which keep their semi-major axis in the $\mathrm{HZ}$ despite the presence of a nearby giant planet. We refer to this value as $f_{\text {hab, } 1 \mathrm{P}}$.

We place 100 test particles in the $\mathrm{HZ}$ with their semi-major axes distributed uniformly from inner to outer edge. We place them 
Table 2. The stellar parameters used to determine the habitable zone using the runaway greenhouse and maximum greenhouse parameters from Kopparapu et al. (2014) and the planetary orbits. The effective temperatures, parallaxes and luminosities are all from Gaia DR2 (Gaia Collaboration et al. 2018); the planetary parameters and stellar mass are determined in the references below. The table is ordered by the distance to the midpoint of the habitable zone.

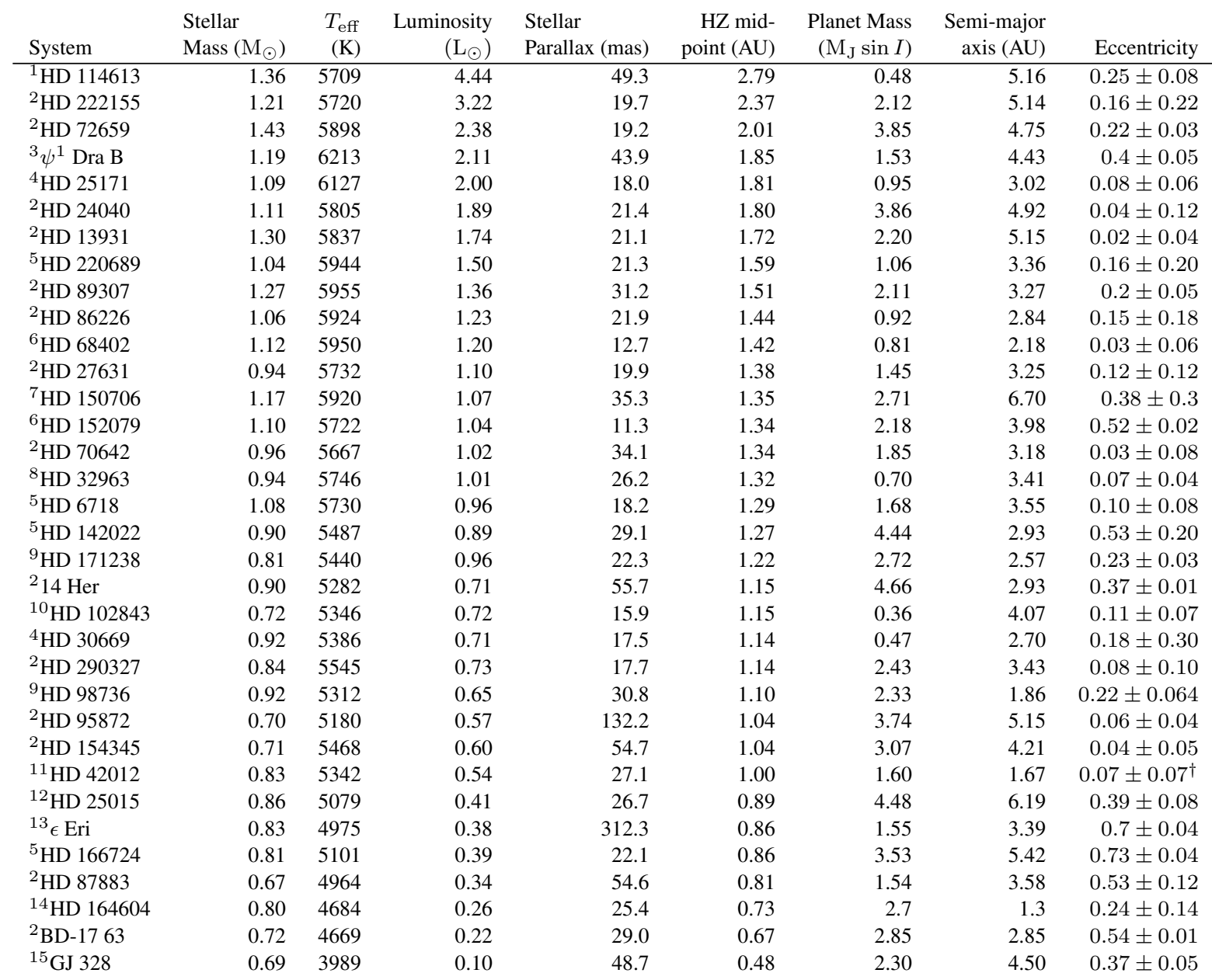

${ }^{1}$ Wittenmyer et al. (2014), ${ }^{2}$ Stassun et al. (2017), ${ }^{3}$ Endl et al. (2016), ${ }^{4}$ Moutou et al. (2011), ${ }^{5}$ Marmier et al. (2013), ${ }^{6}$ Jenkins et al. (2017), ${ }^{7}$ Boisse et al. (2012), ${ }^{8}$ Rowan et al. (2016), ${ }^{9}$ Ment et al. (2018), ${ }^{10}$ Feng et al. (2019) ${ }^{11}$ Rey et al. (2017), ${ }^{12}$ Rickman et al. (2019), ${ }^{13}$ Benedict et al. (2006) ${ }^{14}$ Robertson et al. (2013),

${ }^{\dagger}$ Note: HD 42012 is given with a $3 \sigma$ upper bound of 0.2 . We reformulate it as in the table to make consistent with the rest of the systems.

on circular orbits and make them co-planar with the planet. We randomly pick the three Keplerian angles (argument of perihelion, $\omega$; longitude of ascending node, $\Omega$, and mean anomaly, $M$ ) between 0 and $360^{\circ}$ and then integrate the systems for $10^{7}$ years. For each system we check if $10 \mathrm{Myr}$ was sufficient for it to relax, i.e. if after $10 \mathrm{Myr}$ particles are still being lost. If any test particle was lost within the last Myr we run the system for another Myr and check again repeating the process until no more test particles are lost. We find that for most systems $10 \mathrm{Myr}$ is sufficient. For each system we do three simulations. One simulation using the reported eccentricity of the giant planets and two using the $\pm 1 \sigma$ eccentricity-values shown in table 2.

The systems we simulate are shown in figure 3 . They are split into three groups:

Group 1: Systems where the planet has a considerably larger semi-major axis than the outer edge of the $\mathrm{HZ}$ and a low eccentricity, or has a moderate eccentricity with a much larger semi-major axis.
Group 2: Systems where the planet has a semi-major axis just outside the outer edge of the $\mathrm{HZ}$ with a low to moderate eccentricity or at larger semi-major axis with a sufficiently high eccentricity to bring the pericentre very close to the HZ.

Group 3: Systems that could fit in either Group 1 or 2, due to the planets' having large uncertainties in their eccentricities.

\subsection{Resilient habitability}

\subsubsection{Planets}

To determine the the resilient habitability we need to perform simulations that can represent the past dynamical evolution of an observed system. We postulate that any simulation of the system's past that ends with a massive planet having an eccentricity consistent with observations can be used to represent the system's history.

Figure 1 shows that the outcomes of planet-planet scattering between planets of two different mass ratios (1:1 and 3:10) covers the entire range of observed eccentricities. We take these two mass 

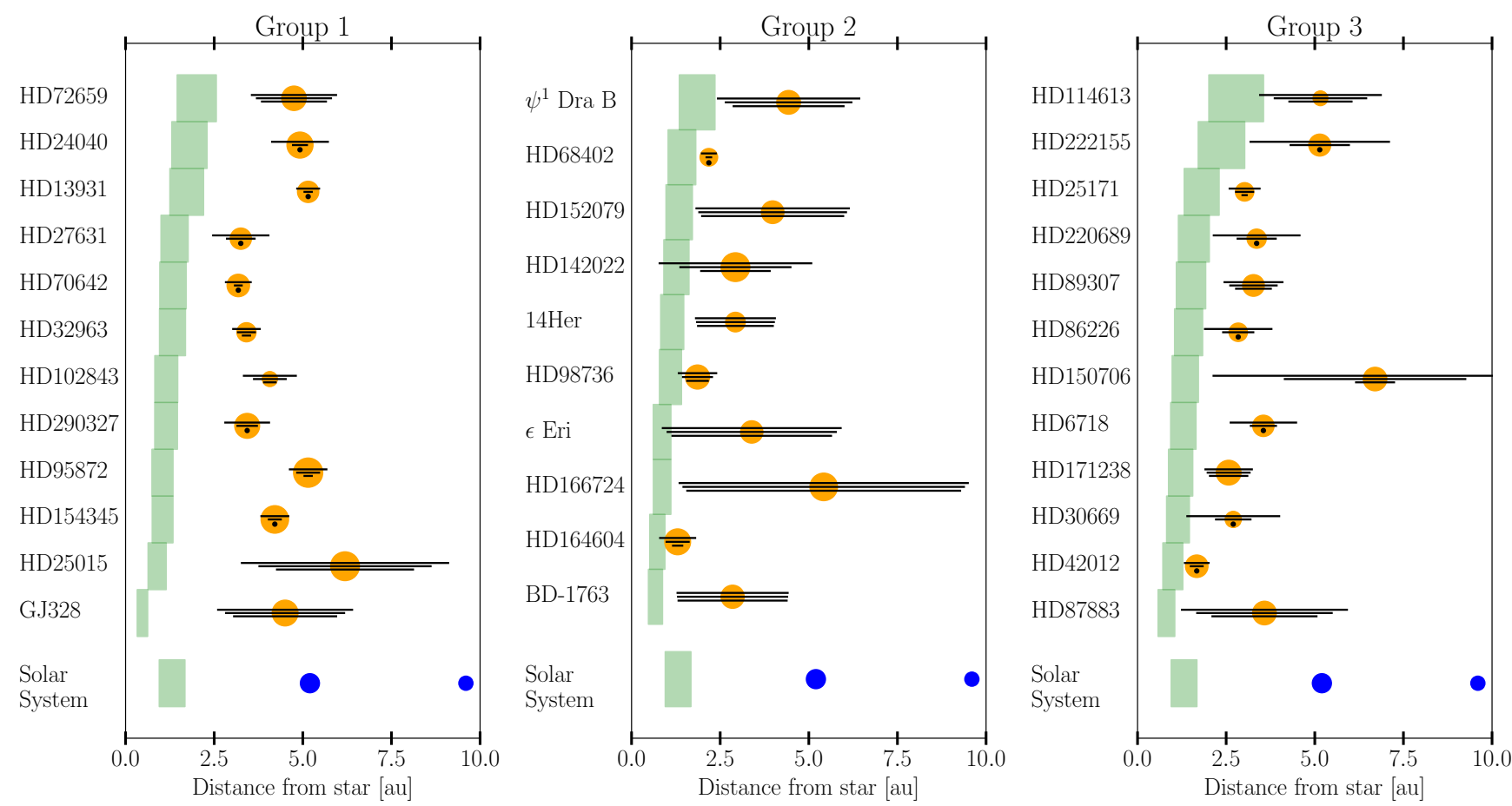

Figure 3. The planetary systems studied in our simulations. The planet is shown as an orange circle, where the area of the circle is proportional to $\sqrt{M_{\mathrm{p}} / M_{\star}}$. The black lines show the peri- and apo-centre range of the orbit using the reported and $\pm 1 \sigma$ values of the eccentricity. If the lower limit is consistent with zero, this is instead represented by a black dot. The green shaded area is the habitable zone calculated according to the formula from Kopparapu et al. (2014) (Equations 1 and 2 of this paper). The systems are split into three categories, from left to right: Group 1 comprises systems with planets on distant orbits and/or with low eccentricity; Group 2 comprises systems with planets on nearby orbits and/or with high eccentricity; and Group 3 comprises systems where the planetary orbits have uncertain eccentricities that could place the systems in either Group 1 or Group 2. The Solar System is shown for scale at the bottom of each panel.

ratios and construct two sets of initial conditions that contain planets with those ratios plus additional ones in order to capture secular effects during and after scattering. We call them $3 \mathrm{E}$ and $4 \mathrm{H}$ and they are set up as follows:

3E: This set contains three equal-mass planets, all with a mass equal to the $M_{\mathrm{p}} \sin I_{\mathrm{p}}$ of the observed planet.

4H: This set contains four planets with hierarchical masses, following the Solar System giants, with the innermost planet having a mass equal to the $M_{\mathrm{p}} \sin I_{\mathrm{p}}$ of the observed planet. We will refer to planets in this set as [lower case] jupiter, saturn, uranus, neptune.

We randomly pick the three Keplerian angles uniformly between 0 and $360^{\circ}$ and draw random initial eccentricities uniformly from 0 to 0.01 . The initial inclinations are drawn randomly between 0 and $5^{\circ}$ which results in mutual inclinations between 0 and $10^{\circ}$ with an average around $2-3^{\circ}$ : this is consistent with observations of Kepler multiple systems (Lissauer et al. 2011; Johansen et al. 2012). The planet radius is set following Bashi et al. (2017). Planets below $0.39 \mathrm{M}_{\mathrm{J}}$ follow a relation of $R \propto M^{0.55}$ and planets with masses above it follow $R \propto M^{0.01}$ with the breakpoint radius being $1.1 \mathrm{R}_{\mathrm{J}}$.

For each set in each system we place the innermost planet at two times the observed semi-major axis and initial separation between the planets is set to 3 mutual Hill radii in the $3 \mathrm{E}$ runs and 3.5 mutual hill radii for the $4 \mathrm{H}$ runs. The mutual Hill radius of two planets with masses $M_{1}$ and $M_{2}$ orbiting a star with mass $M_{\star}$ at semi-major axes of $a_{1}$ and $a_{2}$ is defined as:

$R_{\mathrm{MHill}}=\left(\frac{M_{1}+M_{2}}{3 M_{\star}}\right)^{1 / 3} \frac{a_{1}+a_{2}}{2}$.

This small spacing is chosen in order to trigger the instability on a short time-scale, and to be computationally efficient. The time until the instability is triggered does not affect the duration of the scattering phase, as that is set by the orbital time-scale of the planets (Malmberg et al. 2011). Nor does it affect broadly the properties of the systems once they have stabilised following the ejection of one or more planets.

\subsubsection{Test particles}

In two-planet scattering one can narrowly constrain the expected range of final semi-major axes of the surviving planet by considering energy conservation (as seen in figure 1). When more planets are added, the final energy of the planets becomes less predictable and the expected range of semi-major axes is widened. Given that we are modelling a system where the planet has a specific semimajor axis we need to circumvent this. We do this by utilizing the fact that planetary dynamics are essentially scale free; i.e. if one takes a system and doubles all the semi-major axes it would almost always have the same exact dynamical outcome. The one exception is the probability of collisions, which decreases with increasing semi-major axis (Ford \& Rasio 2008). We find them to be rare in our simulations $(<3 \%)$ so this is not a concern. Given this, we can rescale the semi-major axes of the remaining planets and the 
location of $\mathrm{HZ}$ according to the semi-major axis of the planet in the system we are modelling.

The post facto rescaling means that a larger range of test particles has to be included in the simulation. We place approximately 450 test particles (circular, coplanar and randomly phased) between 0.8 times the inner edge of the $\mathrm{HZ}$ to 2.5 times the outer edge. The exact number is determined so the spacing between them is the same as in the previous experiment. Any realization (run) that ends with the rescaled location of the $\mathrm{HZ}$ outside where initially there were test particles is discarded.

Two changes are made to the simulation to ensure efficiency. Firstly, the ejection distance is set to 250 au which ensures that test particles which clearly are not going to be habitable are removed in a timely fashion. Second, we we remove any test particles that pass within $0.05 \mathrm{au}$ of the host star. This removes highly eccentric test particles that would significantly slow down the integration during their pericentre passages.

We then run the simulations for $10^{7}$ years. Our initial conditions trigger the instability in less than $10^{5}$ years in all systems and the scattering phase rarely lasts more than $10^{6}$ years. This means that $10^{7}$ years is usually more than enough for the system to evolve and the phase of scattering to end. However if there are test particles being ejected in the last Myr we run the simulation until there has been a Myr with no ejections.

The resilient habitability for each run is calculated by rescaling the location of the HZ by $a_{\text {final }} / a_{\text {obs }}$, checking which particles started with their semi-major axes in it and then seeing what fraction are left at the end.

\section{RESULTS}

As explained earlier, we considered the dynamical evolution of two kinds of planetary systems: 3E (where we have three planets of the same mass) and $4 \mathrm{H}$ (where we have four planets following the same ratio of masses of our own gas giants). For each system we investigated, we ran 100 simulations for each architecture following a phase of instability and planetary scattering: this ejected some planets and left the inner planet on a more eccentric orbit. In Section 2, we showed that scattering of a Jupiter and a Jupiter, and of a Jupiter and a Saturn, can between them span the observed eccentricity range. Therefore, we first consider the question: what blend of $3 E$ and $4 H$ runs best matches the observed eccentricity distribution of all observed giant exoplanets?

We determine the weighting by comparing the eccentricity distribution we find for the innermost planets in our simulations with observations. Our observational sample is taken from the exoplanet archive ${ }^{2}$. We select giant planets with masses in the range $[0.3,8] \mathrm{M}_{\mathrm{J}}$ and semi-major axes $a>1.3$ au orbiting stars with masses $[0.65,1.4] \mathrm{M}_{\odot}$ resulting in a sample of 177 planets.

We generate 1000 eccentricity distributions for a range of $3 \mathrm{E}: 4 \mathrm{H}$ ratios from the simulated eccentricities by randomly sampling them without replacement. We then perform a 2-sample KStest between the observed eccentricities and our generated distribution. We find that randomly drawn distributions with $3 \mathrm{E}: 4 \mathrm{H}$ ratios between 2:3 and 5:4 are consistent with the observations in more than $95 \%$ of the cases, and we chose to use a 1:1 ratio. The comparison is shown in figure 4 , and the resulting fraction of initially $4 \mathrm{H}$ systems as a function of eccentricity is shown in figure 5. Figure 5

2 https://exoplanetarchive.ipac.caltech.edu/ as of February 11th 2019

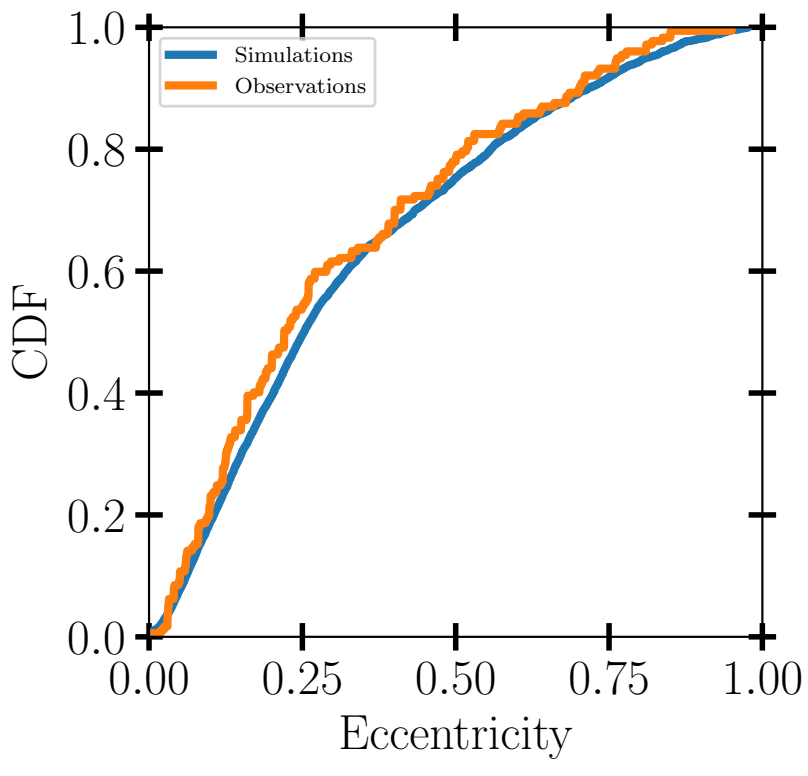

Figure 4. The cumulative distribution of exoplanet eccentricities. The observed RV sample is shown in orange. In blue we show the distribution arising from a 1:1 blend of the results of our two sets of simulations, $3 \mathrm{E}$ and $4 \mathrm{H}$. For the $3 \mathrm{E}$ simulations we use the eccentricity of the innermost planet, while for the $4 \mathrm{H}$ simulations we use the eccentricity of jupiter which in $\sim 97 \%$ of the cases is also the innermost planet.

can also be interpreted as the probability of a system with a given reported eccentricity to initially have been a $4 \mathrm{H}$ system.

For each of the systems illustrated in figure 3, we modelled dynamical histories using both the $3 \mathrm{E}$ (three equal-mass planets) and $4 \mathrm{H}$ (four planets following the same ratio of masses of our own gas giants). For each system, we performed 100 runs for each of the $3 \mathrm{E}$ and $4 \mathrm{H}$ architectures. For further analysis, keeping those where the inner planet's orbital eccentricity matched that of the observed system (within the $1 \sigma$ uncertainties for eccentricity).

We used the distribution shown in figure 5 to weight the $3 \mathrm{E}$ and $4 \mathrm{H}$ runs for each system to determine its $f_{\text {rhab-distribution. Of- }}$ ten, the number of $3 \mathrm{E}$ and $4 \mathrm{H}$ runs that fall within the eccentricity range for a system do not match the expected fraction from figure 5 , given its eccentricity. This is because when determining the expected fraction as a function of the reported eccentricity we use the eccentricities at the end of our simulations $\left(e_{\text {final }}\right)$, whereas we allow for eccentricity oscillations (i.e. angular momentum exchange via secular interactions) when determining the $f_{\text {rhab-distribution. }}$ We allow for the oscillations because a number of runs would have ended up in the correct eccentricity range if the simulation had been stopped at a different time. After taking this into account we upsample either the included $3 \mathrm{E}$ or $4 \mathrm{H}$ runs to give the correct ratio for the reported eccentricity of the observed system. Once we know which runs to use and how to re-sample them we determine the $f_{\text {rhab-distribution. }}$

Figure 6 shows the outcome of a subset of runs simulating HD 72659. The resilient habitability, i.e. the fraction of the test particles originally in the habitable zone (HZ) remaining within the $\mathrm{HZ}$ at the end of the run, $f_{\text {rhab }}$, for each run is shown in the panel on the right. The figure includes a correctly-weighted combination of $4 \mathrm{H}$ and $3 \mathrm{E}$ runs, differentiated by the colour of the $\mathrm{HZ}$ where green represents $4 \mathrm{H}$ and light blue $3 \mathrm{E}$. All of the runs have eccentricities consistent with the reported eccentricity, when we include 


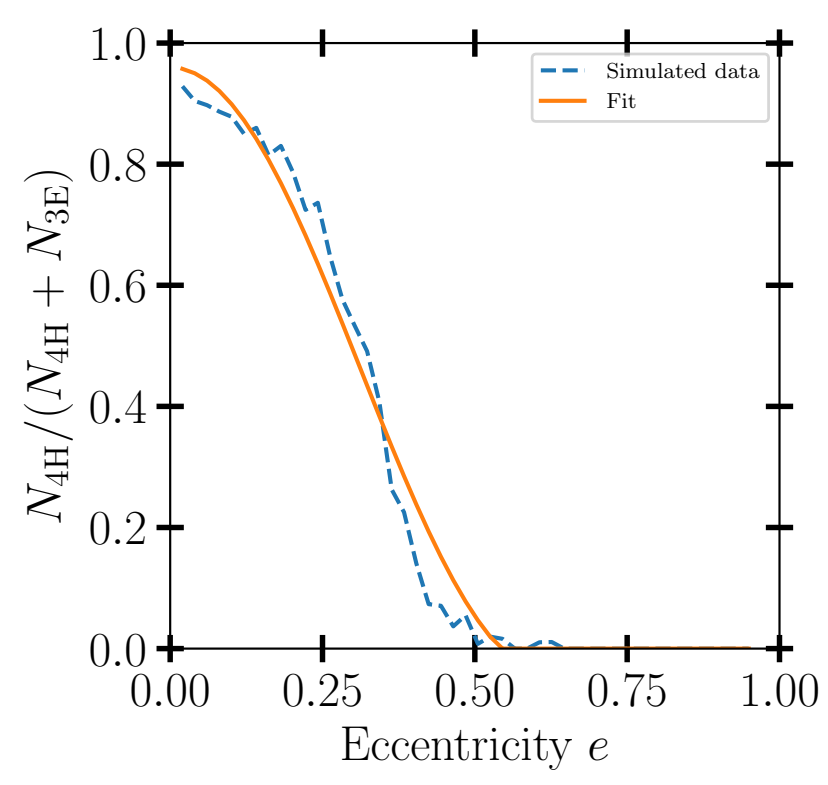

Figure 5. Here we show the fraction of initially $4 \mathrm{H}$ systems as a function of final eccentricity, when using the 1:1 global blend shown in Figure 4. The blue dashed line shows the results from the simulations, while the solid orange line shows a polynomial fit.

secular eccentricity oscillations as described above. We show the configurations of the remaining planets for each of 48 runs, sorted in decreasing $f_{\text {rhab }}$. Additionally we show at the top the initial configurations for $3 \mathrm{E}$ and $4 \mathrm{H}$. The colour labelling for the initial configuration and the final 48 systems is the same. For each planet, the location of the circle denotes the semi-major axis whilst the error bars denote the maximum and minimum distances form the host star. The light backgrounds show the range of the giant planet semi-major axes (light blue) or positions (light red) over the course of each run, sampled every $10 \mathrm{kyr}$.

We see a clear difference between the $3 \mathrm{E}$ and $4 \mathrm{H}$ runs: $4 \mathrm{H}$ gives a broad range of $f_{\text {rhab-values, even when looking at runs }}$ with similar eccentricities: while the upper quartile have $f_{\text {rhab }} \approx$ $0.75-1$, others show much smaller values of $f_{\text {rhab }}$. On the other hand, for the $3 \mathrm{E}$ runs we see that in most cases $f_{\text {rhab }} \approx 0$; we see that the vast majority of the twelve $3 \mathrm{E}$ runs shown are located at the bottom of the distribution. Massive planets have directly invaded the $\mathrm{HZ}$ in about one half of the $3 \mathrm{E}$ runs, leading in all these cases to the essentially complete removal of test particles. However, we see a few examples of $4 \mathrm{H}$ runs where the $\mathrm{HZ}$ is invaded without equally destructive outcomes. This is because the planet in question was of much lower mass (roughly that of Neptune). In a large fraction of runs we can see that test particles have been ejected even though a planet never entered the HZ. This, along with the gaps seen in the test particle distribution in nearly every run, can be explained by resonances which are pumping up the eccentricities of the test particles. A detailed discussion of the resonances will follow in section 6.1.

\section{1 $f_{\text {rhab }}$ and $f_{\text {hab, } 1 \mathrm{p}}$ distributions for the three groups}

As can be seen in figure 6, runs for a given system can show a very broad range of values of $f_{\text {rhab }}$. Therefore for each system, we plot the range of values of $f_{\text {rhab }}$ (the $1 \sigma$-equivalent range, i.e., the central $68 \%$ of the distribution) in figure 7 , together with the present day habitability simulations, $f_{\text {hab,1P }}$, in black. The systems are separated into the three Groups as shown in figure 3. We consider the results of each Group in turn.

\subsubsection{Group 1 systems}

Group 1 (left panel in figure 7) contains systems where the planet has a considerably larger semi-major axis than the outer edge of the $\mathrm{HZ}$ and a low eccentricity, or has a moderate eccentricity with a much larger semi-major axis. All of the systems show high values of $f_{\text {hab }, 1 \mathrm{P}}$ : usually essentially unity. In all cases the mean value of $f_{\text {rhab }}$ is lower and the distribution shows a broader range of values.

For the three systems with higher eccentricities (HD 72659, GJ 328 and HD 25015) the width of the distribution can be understood by considering figures 5 and 6 . The higher the observed eccentricity, the larger the fraction of $3 \mathrm{E}$ runs contributing to the

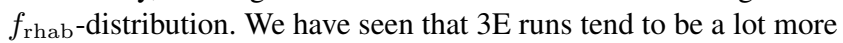
disruptive to test particle orbits. However, GJ 328 and HD 25015 differ from all the other Group 1 systems in that the giant is considerably more distant from the HZ, resulting in nearly all of the $4 \mathrm{H}$ runs along with a significant fraction of the $3 \mathrm{E}$ runs giving $f_{\text {rhab }} \approx 0.9$

The other systems in Group 1 are all at low eccentricities and

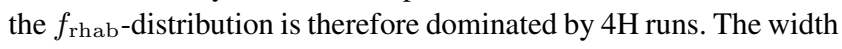
of the $1 \sigma$-range comes from the diversity of outcomes of the $4 \mathrm{H}$ runs, which can be seen in figure 6 . More specifically, for the $4 \mathrm{H}$ runs we find that $40 \%$ of the runs have $f_{\text {rhab }} \approx 1$ with the rest of them fairly evenly spread between 0 and 1 . Figure 8 shows the distribution of $e_{\text {final }}$ and $f_{\text {rhab }}$ for all the runs. We see that low eccentricities have a mix of low and high values of $f_{\text {rhab }}$ whilst eccentricities above 0.5 give only very low values of $f_{\text {rhab }}$. Some $17 \%$ of runs yield $f_{\text {rhab }}>0.9$ whilst $46 \%$ of runs result in $f_{\text {rhab }}<0.1$. The distribution for an individual system can often deviate from the average of all runs shown here. The eccentricity at which the peaks appear is set by the underlying eccentricity distribution resulting from the planet-planet scattering. A larger planet/star mass ratio shifts the peaks to higher eccentricities. The relative strength of each peak is set by the distance between the $\mathrm{HZ}$ and planet in terms of Hill radii. $4 \mathrm{H}$ runs in systems with distant planets such as HD 95872 nearly always have $f_{\text {rhab }}>0.95$ and for the $3 \mathrm{E}$ runs such systems (and only those) have a fraction of runs showing high survival. Systems where the planet sits close to the HZ like HD 72659 do the opposite. The $4 \mathrm{H}$ runs are distributed fairly evenly between $f_{\text {rhab }}=0$ and $f_{\text {rhab }}=0.95$ and the $3 \mathrm{E}$ runs are always fully destructive.

\subsubsection{Group 2 systems}

Group 2 (middle panel in figure 7) contains systems where the planet has a semi-major axis just outside the outer edge of the $\mathrm{HZ}$ with a low to moderate eccentricity, or has a larger semi-major axis with a sufficiently high eccentricity to bring the pericentre very close to the HZ. Such planets are more destructive to objects within the HZ. Other than HD 68402 all of the systems have a low $f_{\text {hab,1P }}$

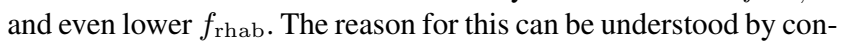
sidering figure 8 . The high eccentricity systems only include runs from a region in the plot where there are no runs with high $f_{\text {rhab }}$, whereas the moderately eccentric ones that sit closer to the HZ have $4 \mathrm{H}$ runs that are shifted to significantly lower $f_{\text {rhab }}$ values as discussed in the previous subsection for the Group 1 systems.

The outlier, HD 68402, is a low-mass giant sitting very close 


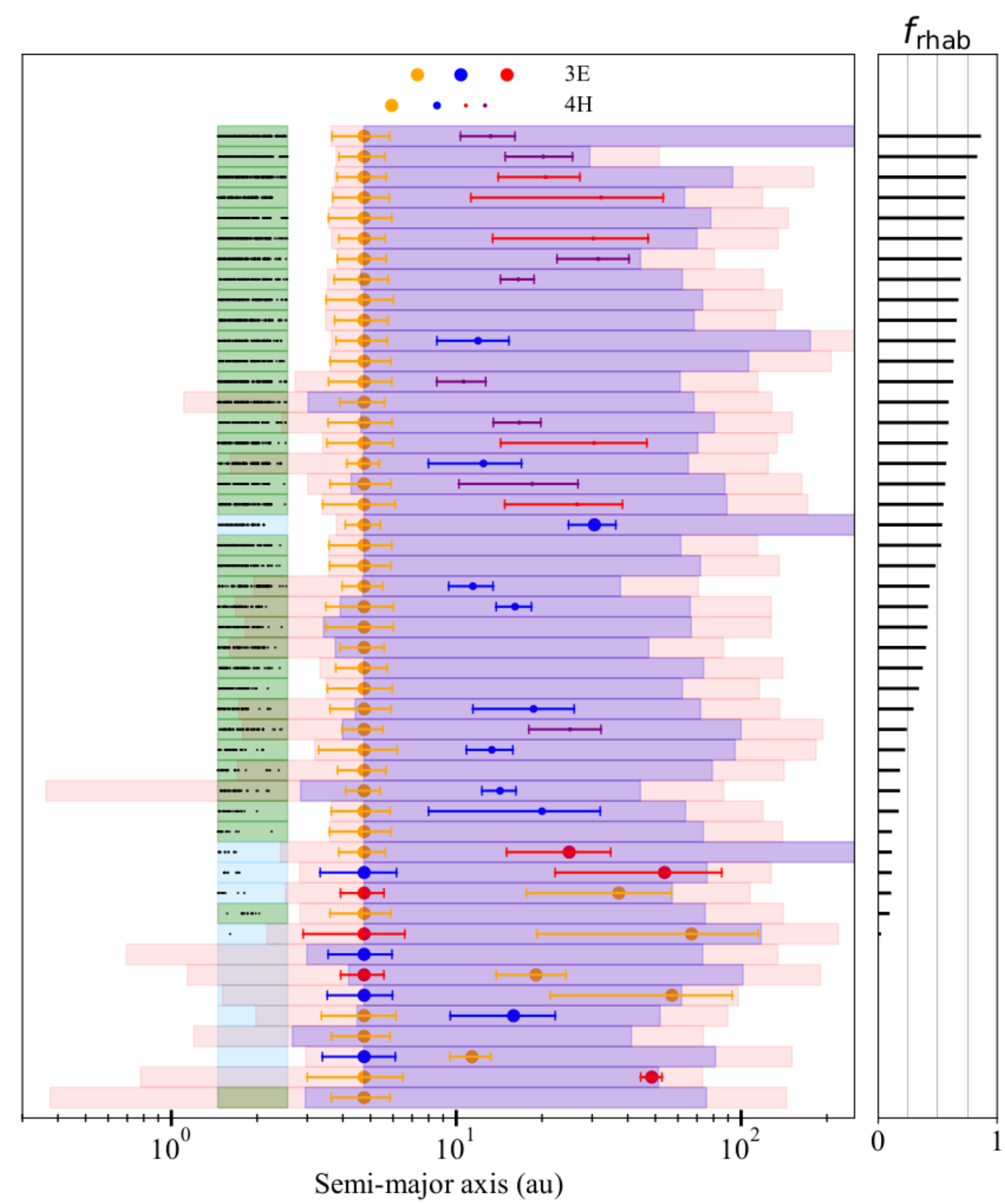

Figure 6. The figure shows the outcome of a set of runs for HD 72659. The habitable zone is shown by the shaded rectangle around 2 au: green for the $4 \mathrm{H}$ runs and cyan for the $3 \mathrm{E}$ runs. Coloured circles show the final semimajor axes of the planets, with the error bars indicating their pericentre and apocentre values. The planets' initial locations, with the same colouration, are shown at the top of the figure. The final semimajor axes of the test particles are shown as small points, blue for those that contribute to $f_{\text {rhab }}$ and grey for those that do not. The shaded blue region shows the range of the planets' semimajor axes during the integration, with output sampled every $10 \mathrm{kyr}$, and the peach region shows the range of the planets' pericentres and apocentres, also sampled every $10 \mathrm{kyr}$. Finally, the panel to the right shows the $f_{\text {rhab }}$ value for each system. 

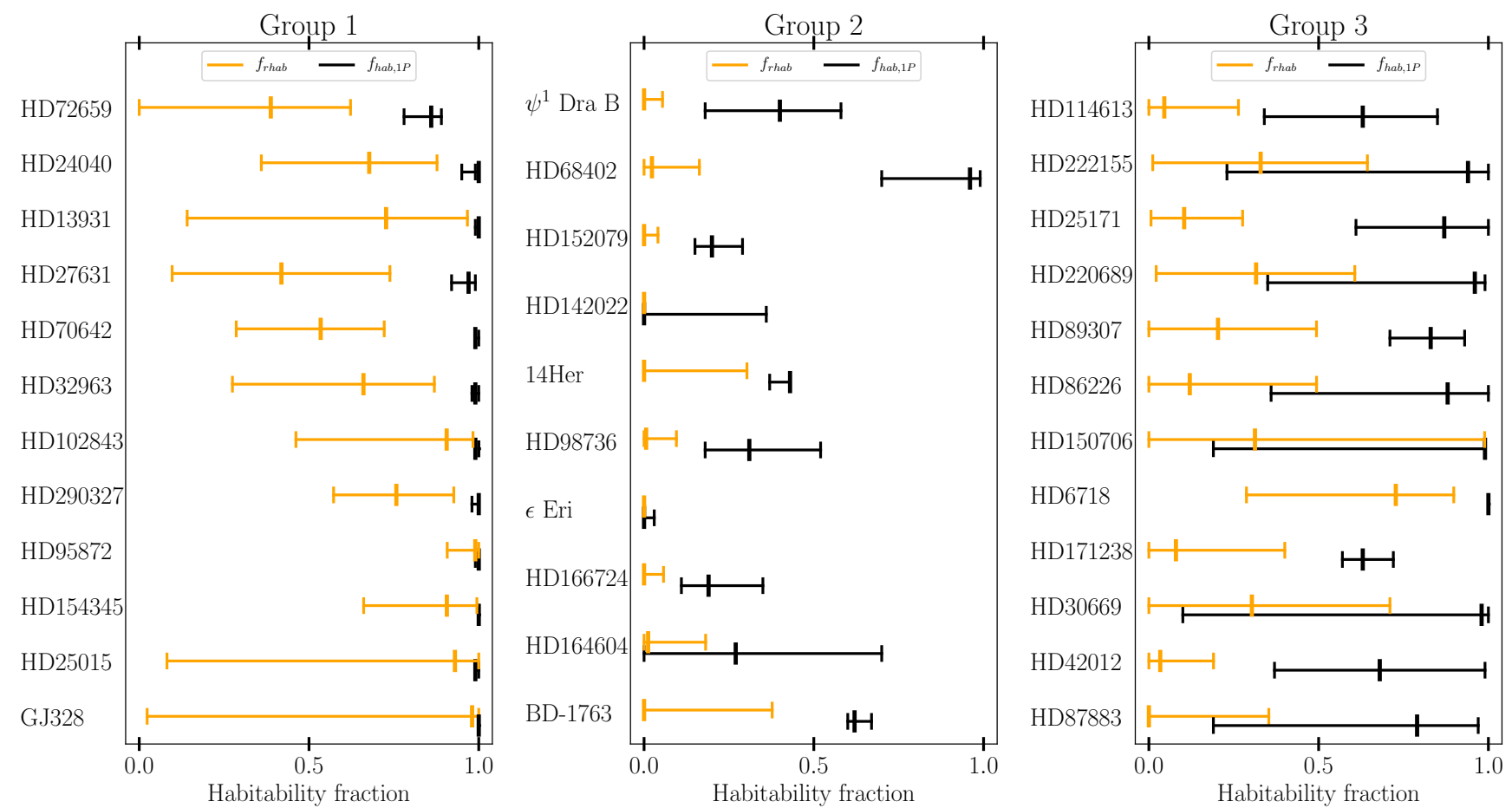

Figure 7. The present day and resilient habitability of all the systems. The black line indicates the present day habitability of the systems, in the configuration shown in figure 3. The midpoint on the line show the survival fraction found when simulating using the reported eccentricity and the endpoints are the result of using $\pm 1 \sigma$ values. The orange line shows the median and " $1 \sigma$ range" (i.e., the central $68 \%$ of the distribution) of the $f_{\text {rhab }}$ distribution for each system, using the blending ratio given by the reported eccentricity (see Figure 5).

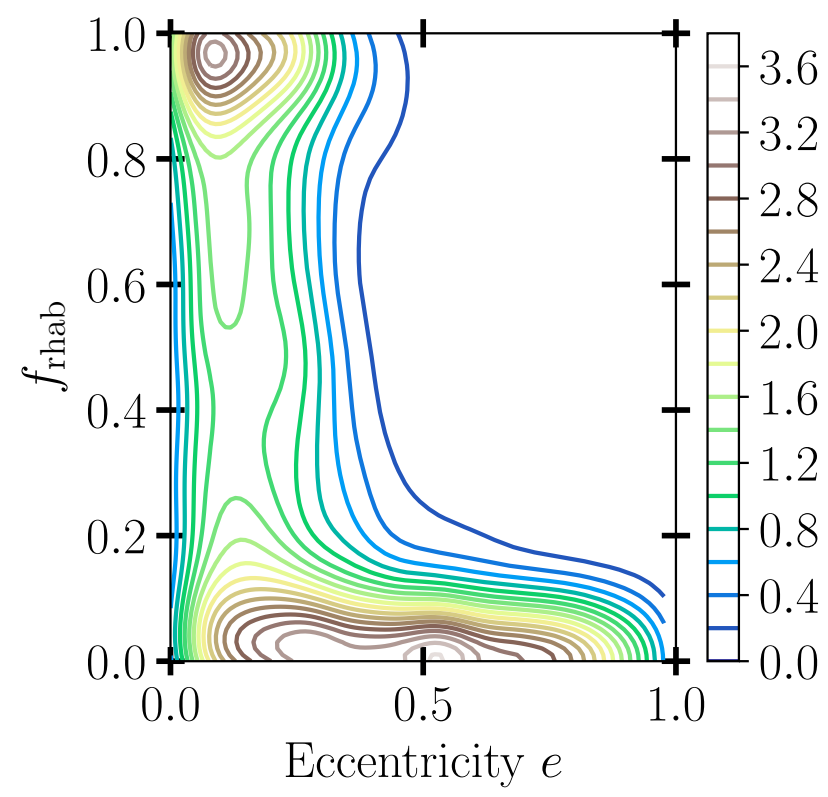

Figure 8. A kernel density estimate showing the resulting $f_{\text {rhab-values for }}$ all our runs, both $3 \mathrm{E}$ and $4 \mathrm{H}$, plotted against the final eccentricity of the planet.

to the $\mathrm{HZ}$ on a nearly circular orbit. In its present day orbit it does very little damage to the $\mathrm{HZ}$; however, during the scattering any eccentricity fluctuations or changes to its semi-major axis easily destabilise test-particle orbits in the HZ. HD 68402 clearly demonstrates how much the history of a system can matter.

\subsubsection{Group 3 systems}

Group 3 contains the systems with a large uncertainty in the reported eccentricity of the planet and could therefore fall in either Group 1 or Group 2. Given that we include runs that are within one standard deviation of the reported eccentricity some time after the final planet ejection occurs, we are including runs from a much wider eccentricity range. We perform a second determination of the $f_{\text {rhab-distribution, in which we pick runs close to the reported }}$ value of the observed eccentricity, and within $0.5 \sigma$ of the $-1 \sigma$ and $+1 \sigma$ values (orange, red and blue colours in figure 9).

The large eccentricity range means that we get a large sample of both $3 \mathrm{E}$ and $4 \mathrm{H}$ runs which widens the distribution. Not all distributions in figure 9 are narrower than the corresponding ones in figure 7; however in a subset of them the majority of $3 \mathrm{E}$ runs get relegated to the high $e$ distribution (blue line in figure 9). This often shifts the other two distributions (orange and red lines) to higher values, in particular for HD 150706. If its actual eccentricity is close to the lower end it is one of the most resilient systems we have looked at. If it is at the high end, the opposite is true. 


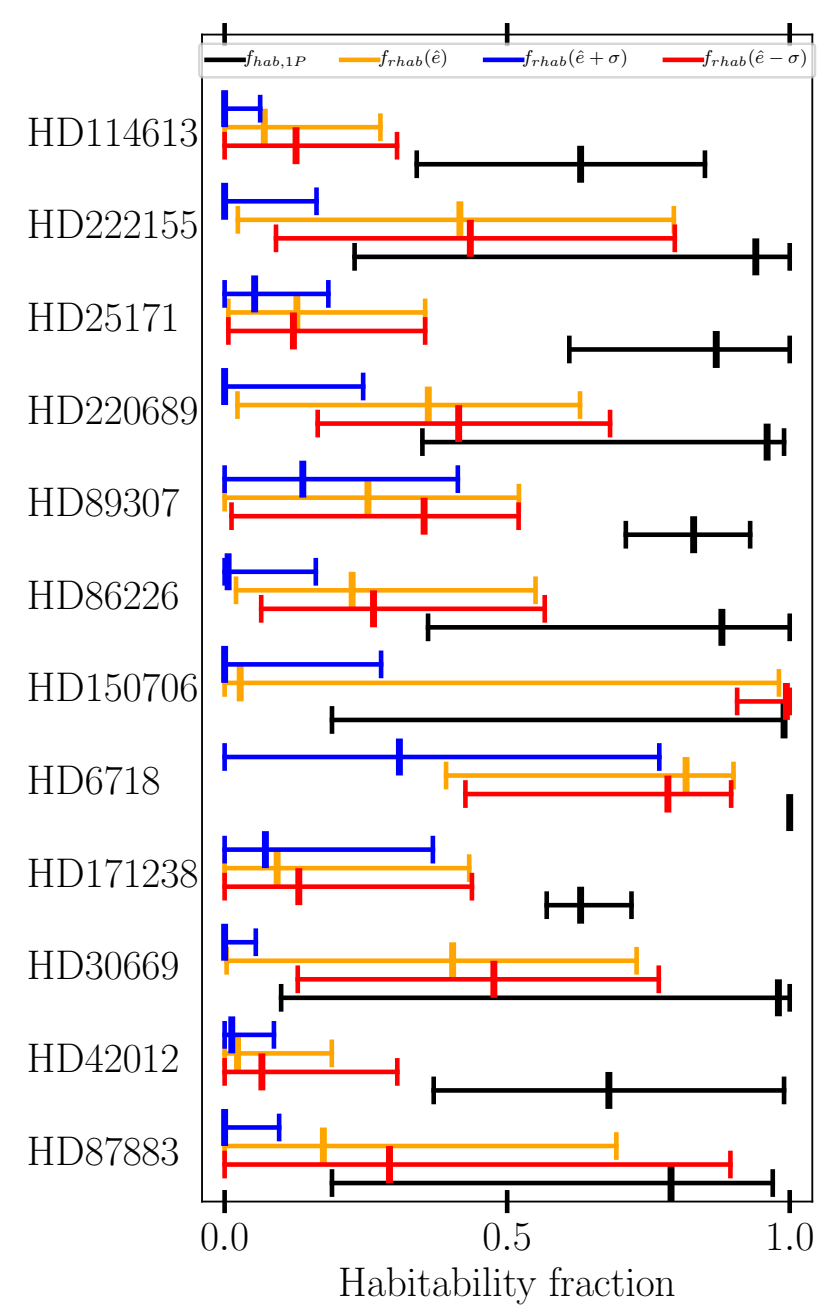

Figure 9. The resilient habitability for systems with large uncertainties in the eccentricity of the gas giant. The three $f_{\text {rhab }}$ distributions are generated using runs close to the $-\sigma$ (red), mean (orange) and $+\sigma$ (blue) values of the eccentricity. The black line shows $f_{\text {hab,1P }}$, as in Figure 7.

\section{DISCUSSION}

\subsection{Mechanisms responsible for the removal of particles from the $\mathrm{HZ}$}

We have shown that particles orbiting in a star's habitable zone can themselves be destabilised during an instability among its giant planets. We now look in more detail at the physical mechanisms responsible for removing particles from the HZ. This differs depending on whether the outer system is an equal-mass $3 \mathrm{E}$ configuration, or a hierarchical $4 \mathrm{H}$ configuration.

The $3 \mathrm{E}$ runs frequently give $f_{\text {rhab }} \sim 0$ as a $\sim$ Jupiter-mass planet is often scattered into the HZ. This results in most test particles being directly gravitationally scattered and then ejected from the system: around $80 \%$ of the test particles removed from the $\mathrm{HZ}$ in the $3 \mathrm{E}$ runs have a close encounter in the $\mathrm{HZ}$ with one or more planets.

In contrast, in the $4 \mathrm{H}$ runs jupiter only ever enters the $\mathrm{HZ}$ in systems where it is initially located very close to it. However, we still see $f_{\text {rhab }} \approx 0$ runs in systems where this is not the case. This happens in one of three ways:
- Saturn gets scattered into a HZ crossing orbit and ejects most of the test particles (rare).

- Uranus or neptune gets scattered into a $\mathrm{HZ}$ crossing orbit and stays there for some time. This increases the eccentricity of the test particles which are later ejected by jupiter (less rare).

- Resonances from jupiter inside the HZ excite the eccentricities of test particles which are later ejected by jupiter (common).

Examining the close encounter history of the test particles, we find that $60 \%$ of all the ejected test particles in the $4 \mathrm{H}$ runs never experience a close encounter with a planet that takes places within the HZ. Rather, their eccentricities are excited by orbital resonances with the giant planets, after which they are typically removed by gravitational scattering once their apocentre brings them close to jupiter. Matsumura et al. (2013) and Carrera et al. (2016) identified secular resonances as being of importance in pumping up particle eccentricities during scattering amongst outer giant planets. Here, we show that for our systems mean-motion resonances (MMRs) with jupiter can be more significant. We discuss the effects of secular resonances in Section 6.2.

MMRs occur when the orbital periods of two bodies lie close to a ratio of integers. Here we consider $1: m$ and $2: m$ resonances between a test particle in the $\mathrm{HZ}$ and the jupiter. These occur where the semimajor axis of the particle in the HZ lies at $a=(1 / m)^{2 / 3} a_{\mathrm{J}}$ or $a=(2 / m)^{2 / 3} a_{\mathrm{J}}$, where $a_{\mathrm{J}}$ is the semimajor axis of the jupiter. Therefore, as the jupiter's semimajor axis moves erratically inwards as it scatters and ejects the outer planets, the mean motion resonances jump through the habitable zone.

We illustrate the effects of the passage of MMRs by comparing two runs from our $4 \mathrm{H}$ simulations of HD 72659. One run has a

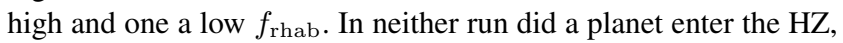
and both end with the jupiter possessing a similar eccentricity.

The motion of the resonances in these two runs is shown in Figure 10. Here the final location of the planet is shown in amber, and the $\mathrm{HZ}$ in green. Vertical lines mark the locations of MMRs and secular resonances, sampled every $10 \mathrm{kyr}$, with the final locations of the MMRs marked as long vertical lines. The final location of each resonance lies at the innermost edge of its range over the integration, as expected from the net inward motion of the jupiter.

In the upper panel of Figure 10, we illustrate a case where the jupiter moves inwards through a slow, incremental process. The sampled locations of the resonances lie dense in the habitable zone, meaning that particles at most semimajor axes in the HZ have time for their eccentricities to be highly excited by one or more resonances. Indeed, particles are found surviving the simulation at only a few semimajor axes, mostly towards the inner edge of the HZ. In the bottom panel, in contrast, the jupiter moves inwards more rapidly, meaning that a significant fraction of the $\mathrm{HZ}$ does not become excited by the passage of MMRs, and many particles survive the integration. These two examples suggest a dependence of a system's resilient habitability $f_{\text {rhab }}$ on the nature of the motion of the jupiter.

We have explored a number of different parametrizations to correlate the evolution of jupiter's semi-major axis with $f_{\text {rhab }}$. We find that looking at the fractional absolute change in semimajor axis between each output time ( $10 \mathrm{kyr}$ resolution) shows this dependence best. The fractional absolute change is given in the form

$\frac{\Delta a}{a}=\sum_{i} \frac{\left|a_{i+1}-a_{i}\right|}{a_{i}}$,

where $a_{i}$ is the semi-major axis at the $i$ th output. Thus, a jupiter that attains its final semimajor axis in a few large jumps will have 

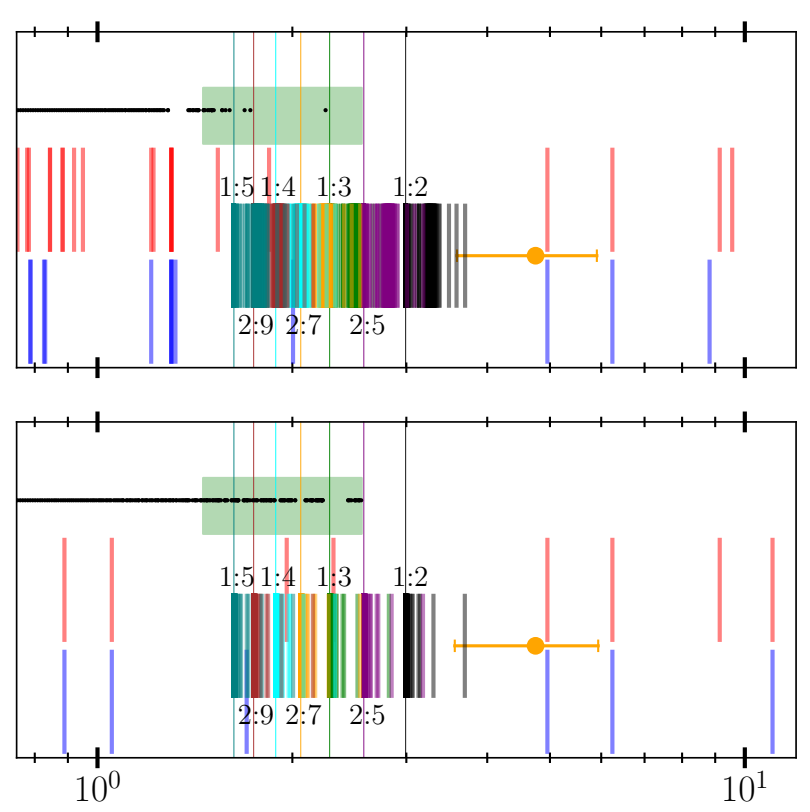

Distance from star [au]

Figure 10. The figures show how the location of orbital resonances changes with time for two different $4 \mathrm{H}$ runs of HD 72659. The final location of the planet is shown in amber, the circle marking its semimajor axis and the error bars its pericentre and apocentre; the habitable zone is shown in green, and surviving test particles as small black points. Vertical lines mark the location of orbital resonances, sampled every $10 \mathrm{kyr}$. The off-axis red and blue lines show the location of the secular eccentricity and inclination resonances. The remaining coloured lines show the $1: m$ and $2: m$ mean motion resonances with the long line being placed at the final location of the resonance.

a small $\Delta a / a$, while those that move in many small steps, which often involves temporary reversals of direction, will have a large $\Delta a / a$. We plot in Figure 11 the resilient habitability for HD 72659 against $\Delta a / a$, for all $4 \mathrm{H}$ runs where no planet entered the HZ. As expected, we see an anticorrelation between $f_{\text {rhab }}$ and $\Delta a / a$.

Combining the anticorrelation we see in figure 11 with the fact that $60 \%$ of ejected test particles in $4 \mathrm{H}$ runs never have a close encounter inside the HZ, we conclude that the majority of test particles in the $4 \mathrm{H}$ case are ejected due to MMRs sweeping over the HZ. The ejection efficiency is correlated with how much the semi-major axis of jupiter changes.

\subsection{Secular resonances in the habitable zone during and after an instability}

One way through which eccentricities can be forced to high values in the absence of direct scattering is through secular resonances. Secular resonances occur when the precession frequency of one of the particles in the HZ matches one of the secular eigenfrequencies of the giant planets. These resonances occur at specific values of the particles' semimajor axis, and formally the forced eccentricity is infinite at these locations. Because the planetary secular frequencies are dependent on their semimajor axes, the frequencies change during planet-planet scattering, and so the secular resonances also move. This process was identified by Matsumura et al. (2013) and Carrera et al. (2016) as a means of destabilising terrestrial planets during an instability amongst giant planets.

Returning to Figure 10, we have plotted the location of the sec-

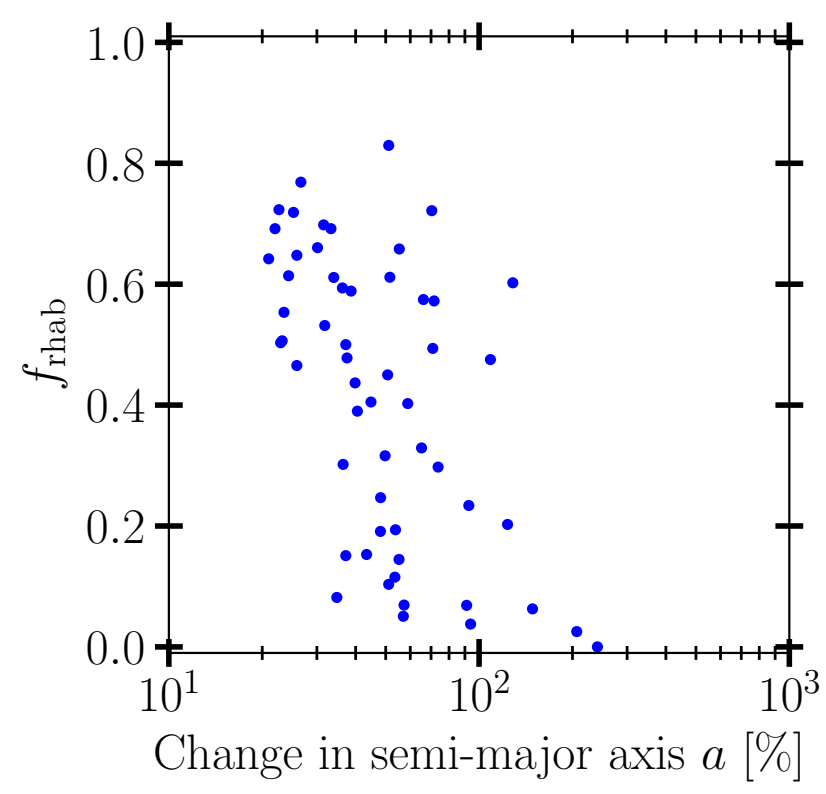

Figure 11. $f_{\text {rhab }}$ as a function of the summed up absolute fractional change in semi-major axis for all 65 of the the 4H-runs of HD 72659 where no planet enters the HZ. The change in semimajor axis is defined by eq 4 .

ular resonances (both eccentricity and inclination) in the two runs every $10 \mathrm{kyr}$. The resonance locations are calculated according to linear Laplace-Lagrange theory (Murray \& Dermott 1999, Ch. 7). They move inwards as the planets' semimajor axes change during scattering, similar to the motion of the MMRs, but in contrast to the MMRs they move much further and more rapidly. This is because they depend on the semimajor axes of all planets, not just the jupiter; but the jupiter alone sets the location of the MMRs and itself moves comparatively little. Indeed, the secular resonances pass across the whole $\mathrm{HZ}$ in just a few 10s of kyr, insufficient time to significantly affect particles' eccentricities. Typically the secular resonances pass through the $\mathrm{HZ}$ in less than $30 \mathrm{kyr}$, and only in $\sim 5 \%$ of runs do they spend more than $100 \mathrm{kyr}$ in the HZ. In the upper panel we do, however, see that the secular eccentricity and inclination resonances remain for several 10s of kyr just interior to the $\mathrm{HZ}$, where they destabilise a few bodies and knock a small hole in the distribution of survivors.

While in principle the final giant planet configuration following scattering could leave a secular resonance in the HZ, this is very rare, occurring in $\lesssim 1 \%$ of our runs. The reason is that the planets must be spaced rather close to each other to place a secular resonance in the HZ. We show in Figure 12 where the secular eccentricity resonances would lie in the HD 95872 system if it contained a second giant planet, as a function of this planet's mass and semimajor axis. To place a secular resonance in the $\mathrm{HZ}$ requires a separation of $\sim 4-7$ mutual Hill radii between the planets. These configurations would be stable if the planets' orbits were near-circular, as they satisfy the limit for Hill stability given by Gladman (1993):

$\frac{a_{\text {outer }}-a_{\text {inner }}}{R_{\text {MHill }}}>2 \sqrt{3}$,

where $R_{\mathrm{MHill}}$ is the mutual Hill radius from equation 3 and $a_{\text {outer }}, a_{\text {inner }}$ are the semi-major axes of the planets. We mark this limit in Figure 12 as a solid red line. While the circular configurations would be stable, scattering often leaves planets with sig- 


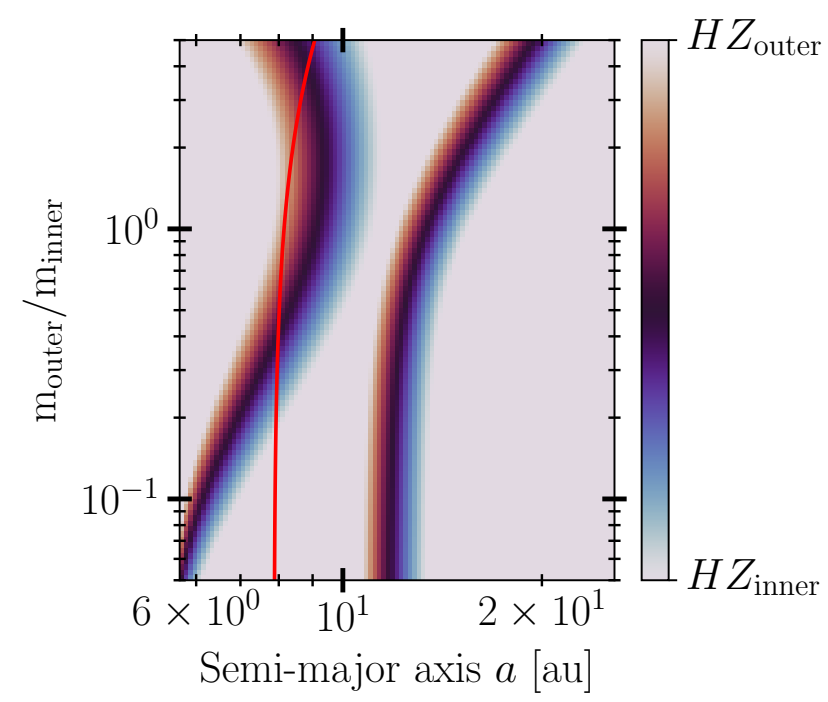

Figure 12. For the system HD $95872(a=4.75 \mathrm{au})$ the figure shows at what semi-major axis a planet of a given mass needs to be placed for either of the secular eccentricity resonances to be in the HZ. The colour indicates where in the HZ the resonance appears. The red line shows the Gladman stability limit for circular orbits: systems wider then this cannot experience orbit-crossing.

nificant eccentricities, and this significantly increases the separation required for the planets to remain stable after scattering (e.g., Mardling \& Aarseth 2001; Mustill \& Wyatt 2012; Giuppone et al. 2013; Petrovich 2015; Antoniadou \& Voyatzis 2016; Hadden \& Lithwick 2018).

\subsection{Low-eccentricity systems}

For the low-eccentricity systems we have to consider whether or not the system has been through a phase of planet-planet scattering, and in either case, what the value of $f_{\text {rhab }}$ will be. If the system has undergone an instability, then the answer is given in figure 7. If there has not been any scattering the only way in which $f_{\text {rhab }}$ could be significantly different from $f_{\text {hab }, 1 \mathrm{P}}$ is if there is an additional hitherto undetected planet in the system. This can affect the system either through changing the eccentricity of the observed planet over time, or by giving rise to secular resonances inside the habitable zone as we have just discussed.

\subsubsection{Eccentricity changing with time}

Secular interactions lead to eccentricities varying with time. These variations occur on timescales of thousands of years, therefore the

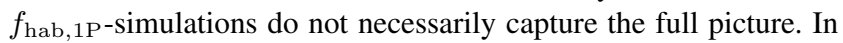
particular, a planet with a low eccentricity at the present day may be at the low point of a secular cycle, if there is an undetected exterior planet currently on an eccentric orbit.

We test how damaging this can be by determining what we call $f_{\text {hab,2P }}$ : here, we take systems where two planets survive our $4 \mathrm{H}$ runs and the jupiter has an eccentricity consistent with the observed system, repopulate the $\mathrm{HZ}$ with low-eccentricity test particles, and run the system for $10 \mathrm{Myr}$. $f_{\text {hab,2P }}$ is then the fraction of these repopulated test particles that survive the integration. By choosing two-planet systems from among our post-scattering configurations, where the outer planet often has a highly-eccentric orbit, we are generating systems where we might expect that a large eccentricity can be passed to the inner planet through secular interactions, and so where $f_{\text {hab,2P }}$ will be low.

However, what we find is that in most cases the variation of the inner planet's eccentricity is smaller than its observational uncertainty, and that the $f_{\text {hab,2P }}$ values lie mostly within the range of $f_{\text {hab }, 1 \mathrm{P}}$ in Figure 7. $f_{\text {hab }, 2 \mathrm{P}}$ is lower if the mean eccentricity of the jupiter over its secular cycles is higher than the reported value, and lower if the reverse is true. In a few percent of cases, the eccentricity of the jupiter at the end of the original integration (the start of the repopulated integration) is close to the minimum or the maximum in its cycle, and in these cases, $f_{\text {hab }, 2 \mathrm{P}}$ can differ significantly from $f_{\text {hab, } 1 \mathrm{P}}$.

We now return to the focus of this subsection: low-eccentricity planets that have not undergone instability. The amplitude of the inner planet's eccentricity oscillations is larger if the outer planet's eccentricity is larger, and so systems that have not undergone instability will usually have values of $f_{\text {hab,2P }}$ similar to their values of $f_{\text {hab,1P, }}$, since this is usually the case for more dynamically excited systems too.

\subsubsection{Placing the secular resonances in the habitable zone}

Nevertheless, there is a potential exception to the fact that systems that have never undergone planet-planet scattering will have a high $f_{\text {hab,2P: }}$ this is if they have secular resonances in the habitable zone. We test the effect such secular resonances can have by setting up runs for the HD 95872 and HD 72659 systems, adding a planet of equal mass on a circular orbit at a semimajor axis that places a secular resonance in the HZ, populating the HZ with test particles, and integrating the system for $10 \mathrm{Myr}$, verifying that the two planets are stable.

For HD 95872 we find that $f_{\text {hab }, 2 \mathrm{P}}$ is as high as $f_{\text {hab,1P }}$ when we place a secular resonance in the HZ; however, a large fraction of the test particles in the $\mathrm{HZ}$ acquire high eccentricities above 0.5 . As we discuss in Section 6.4, these high eccentricities are likely detrimental to any planet's habitability. The secular resonance in this system is especially strong because of the large planet mass relative to the star $\left(3.74 \mathrm{M}_{J}\right.$ versus $\left.0.7 \mathrm{M}_{\odot}\right)$. In HD 72659 , the test particles that have large eccentricities excited eventually get ejected, since the giant planet's pericentre is close to the HZ. HD 95872 and HD 72659 show respectively the highest and the lowest values of both $f_{\text {hab,1P }}$ and $f_{\text {rhab }}$, showing that the effects of secular resonances could be significant for many systems.

As discussed above, it is less likely that systems with higheccentricity planets have secular resonances in the HZ because of the constraints imposed by stability of the gas giants. In answering the question "how likely is a habitable zone to be destabilised by a secular resonance?", we therefore have a curious inverse dependence on eccentricity, where the systems with more eccentric giant planets are less likely to have their $\mathrm{HZ}$ destabilised by a secular resonance. This does, however, ignore the scattering history of the giant planets, which may have destabilised bodies in the $\mathrm{HZ}$ by scattering and the passage of MMRs.

\subsection{The effect of eccentricity on habitability}

The habitable zone is defined as the spherical shell around a star at which the flux received allows for liquid water to exist on the surface of the planet under a set of atmospheric conditions. In our simulations we have hitherto considered only whether the semi- 


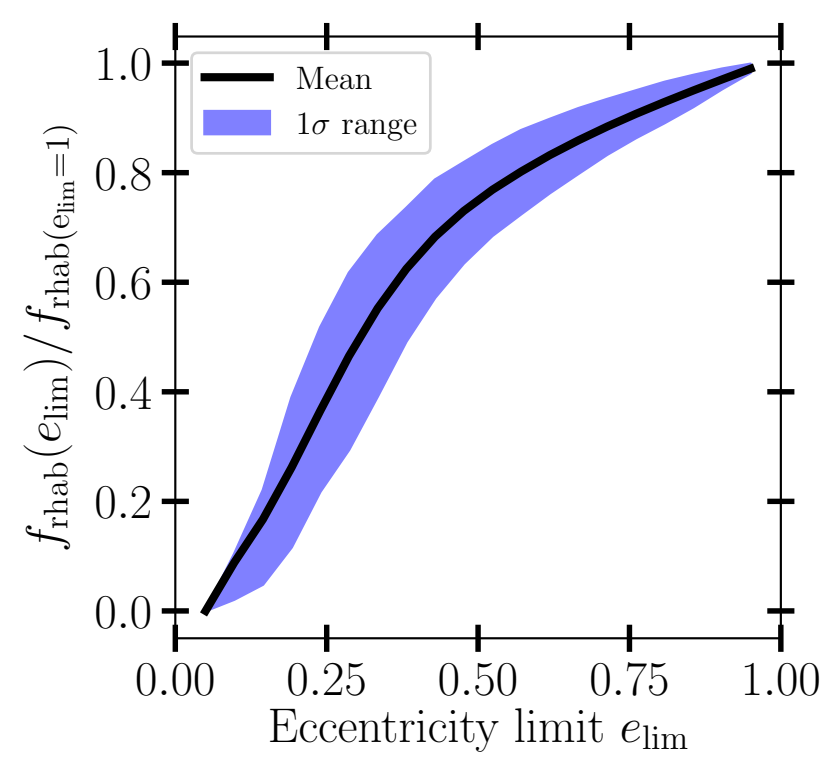

Figure 13. The change in $f_{\text {rhab }}$ for all systems when an eccentricity limit is imposed on the habitability. For each value of $e_{\text {lim }}$ we recalculate $f_{\text {rhab }}$ and do not count any test particle toward it that reaches an eccentricity greater than $e_{\text {lim }}$ throughout the run. The $1 \sigma$ error band shows the standard deviation over all runs of all systems.

major axis remains in the HZ, ignoring any orbital eccentricity the planet may possess.

At some point this approximation breaks down and we have to consider the effects of having large temperature differences between peri- and apo-centre. In general, the higher the orbital eccentricity at a given semimajor axis, the higher the orbit-averaged radiation flux from the star: therefore, the habitable zone is more distant at higher eccentricity. While simple energy-balance arguments give a dependence of the $\mathrm{HZ}$ on eccentricity of $a_{\mathrm{HZ}} \propto\left(1-e^{2}\right)^{-1 / 4}$, more detailed modelling (e.g., Dressing et al. 2010; Spiegel et al. 2010; Linsenmeier et al. 2015; Bolmont et al. 2016; Kane \& Torres 2017), incorporating the effects of planetary spin rate and obliquity, shows that high eccentricities $(e \gtrsim 0.5)$ can have a significantly stronger effect.

As the actual effect of eccentricity depends on other parameters such as the spin rate and obliquity, we explore the number of stable particles that attain a specified value of eccentricity, $e_{\text {lim }}$, at any point in the run. We then recalculate the resilient habitability $f_{\text {rhab }}$ by excluding the particles that reached a high eccentricity, assuming that they have been rendered uninhabitable. By performing this calculation for a range of $e_{\mathrm{lim}}$, we can find how significantly the inclusion of an eccentricity consideration affects $f_{\text {rhab }}$, without committing ourselves to a single (model-dependent) eccentricity limit. We then show in Figure 13 the ratio of $f_{\text {rhab }}$ with the eccentricity limit to that without. As expected, this rises from zero at $e_{\text {lim }}=0$ (all particles are considered uninhabitable when they acquire any eccentricity) to unity at $e_{\lim }=1$ (all surviving particles are considered habitable, regardless of eccentricity). For $e_{\text {lim }}=0.5$, about $30 \%$ of surviving particles are rendered uninhabitable by acquiring eccentricities above $e_{\text {lim }}$. This is a significant reduction in resilient habitability, motivating further studies of the climate of highly-eccentric terrestrial planets.

\subsection{Additional considerations}

\subsubsection{Multiple massive terrestrial planets}

We have used massless test particles to represent terrestrial planets in our simulations. This is computationally efficient as one can simulate many hundreds of test particles within one single run. The total mass of the terrestrial planets is $\lesssim 1 \%$ of that of the giant planets, so using test particles to represent massive terrestrial planets should not be a problem when considering the direct interactions between the particles and the giant planets. However, use of massless non-interacting test particles means that we do not account for any interactions between the habitable planets, should more than one be present.

In principle, if there are multiple terrestrial planets close to each other they can couple to each other and counteract external perturbations. The stabilization by such couplings has been confirmed for both the Solar System (see e.g. Innanen et al. 1997; Batygin \& Laughlin 2008; Laskar \& Gastineau 2009; Zeebe 2015), and generally for tightly packed systems (see e.g. Malmberg et al. 2011; Kaib et al. 2011; Hansen 2017; Mustill et al. 2017; Denham et al. 2019). The key factor determining whether the system is stabilised is the time-scale. If the coupling between the planets is on a short time-scale compared to the perturbation the planets will be resistant to it. Thus, we might expect the resilient habitability of systems to increase if the test particles are replaced with a handful of massive planets.

On the other hand, Carrera et al. (2016) investigated the resilient habitability of systems where the habitable zone was populated either by a swarm of test particles, or by one, two or four massive $\left(1 \mathrm{M}_{\oplus}\right)$ planets. As expected, the survival rate of the single planets is consistent with that of the test particles, but the survival of HZ planets in the systems of two or four terrestrial planets is in fact lower than that of the test particles. In practise then, multiplanet systems subject to strong scattering from an outer system are slightly more vulnerable and more likely to be destabilised, although in systems with weaker external forcing it is likely that the protective coupling can still stabilise the system.

\subsubsection{The timing of the instability}

It is thought that terrestrial planets usually take $10-100$ Myr until they are fully formed. This time-scale is typical in simulations of terrestrial planet formation from swarms of planetary embryos (Chambers 2001; O'Brien et al. 2006; Hansen 2009; Raymond et al. 2009; Lambrechts et al. 2019), and agrees with radiogenic dating of the Earth and Moon (Jacobsen 2005; Halliday 2008). On the other hand, gas giants must form in a few Myr while the gas disc is still present (Machida et al. 2010; Piso \& Youdin 2014; Piso et al. 2015; Bitsch et al. 2019). The instability amongst the giant planets occurs some time after they have formed. It is a steep function of their orbital separation, and the time-scale of onset at a given separation can vary by an order of magnitude (Chambers et al. 1996; Shikita et al. 2010). Therefore, the instability can take place before or after terrestrial planet formation is complete. Indeed, in consistent simulations of formation, migration and dynamics, Bitsch et al. (2019) found that $5 \%$ of the giant planet systems they formed were unstable on timescales $<10 \mathrm{Myr}$.

Thus, depending on the timing of the instability, the terrestrial planets may be fully formed or may still be a swarm of embryos. Whether the test particles in our simulations are considered to represent fully-formed planets or planetary embryos leads to two different interpretations. 
If the instability happens after terrestrial planet formation has finished, then each particle lost from the simulations represents one of a handful of fully-formed planets. Each run can then be interpreted as simulating a large number of different realisations of an inner terrestrial planet subject to the same forcing from the unstable gas giants. Thus, a value of $f_{\text {rhab }}$ of $25 \%$ simply means that on average one quarter of planets would be retained and three quarters lost. If multiple fully-formed terrestrial planets are present in the same system, then their mutual interactions can affect the dynamics: Carrera et al. (2016) showed that this results in a lower fraction of planets surviving.

On the other hand, if the instability occurs before terrestrial planet formation is completed, our test particles can more readily be interpreted as representing the swarm of planetary embryos present in the habitable zone in one system. Thus, a value of $f_{\text {rhab }}$ of $25 \%$ now means that the habitable zone loses three quarters of its embryos. Depending on their distribution and excitation, terrestrial planet formation may not be able to proceed all the way to Earth mass, but may stall at smaller masses as much of the available material can be ejected from the system during the instability. An additional complication concerns the outcome of collisions between embryos: when excited by an instability in the outer system, collision velocities increase and collisions between embryos result in significant mass loss (Mustill et al. 2018). This can reduce the final size of the terrestrial planets still further, if the ejecta is ground down and removed by radiation pressure before being reaccreted by one of the embryos.

\subsubsection{RV mischaracterization of planet eccentricity}

The signal from a planet with a seemingly eccentric orbit may, in some circumstances, actually be generated by a planet on a circular orbit. There are two ways in which this could happen. The first arises from the fact that eccentricity is a positive-definite quantity: it cannot be negative. Therefore, the fitted eccentricities for planets on circular or low-eccentricity orbits are biased towards higher values (Lucy \& Sweeney 1971; Shen \& Turner 2008; Zakamska et al. 2011; Hara et al. 2019). For example, Zakamska et al. (2011) show that the fraction of low eccentricity systems could be underestimated by as much as a factor of 3 . This means that the true values of the resilient habitability for some of our systems may be higher than we have calculated, as the known planets' eccentricities may be somewhat smaller than the reported literature values.

The second way in which a seemingly eccentric signal can be generated by a planet on a circular orbit is through the presence of an interior, lower mass planet at a 2:1 period ratio. Anglada-Escudé et al. (2010) showed that two such planets show a similar and sometimes indistinguishable RV signal as only having the outer, more massive planet on an eccentric orbit. They show that the RV signal from as many as $35 \%$ of observed eccentric single planet systems show no statistically significant difference from being two planets in a 2:1 configuration rather than a single eccentric planet. Further, Wittenmyer et al. (2019) sets an upper bound on the possible apparent eccentricity at 0.5 .

We first make a simple estimation of the destructive effects on the $\mathrm{HZ}$ of a single eccentric planet compared to two planets in a 2:1 configuration. We estimate the mass of the inner companion required to mimic an eccentric orbit from figure 3 of Kürster et al. (2015), and compare the potential for direct scattering in both configurations, comparing the reach of the Hill radius of the inner planet $a_{\mathrm{in}}-r_{\mathrm{H} \text {,in }}$ with that of the seemingly eccentric outer planet at pericentre $a_{\text {out }}\left(1-e_{\text {out }}\right)-r_{\mathrm{H} \text {,out }}$. We find that the eccentric outer planet will be more effective at direct scattering than the 2:1 two-planet configuration.

We test this with a set of simulations and find that systems with $e<0.3$ also can be more damaging than their 2:1 equivalent. This occurs if the pericenter distance to the outer edge of the HZ is smaller than the distance from the extra interior planet to the edge in terms of Hill's radii. This is the case for nearly all systems in this range that are fairly close to the $\mathrm{HZ}$, barring the ones where the 2:1 resonance sits inside the $\mathrm{HZ}$ which already show low $f_{\text {hab,1P }}$ and mostly $f_{\text {rhab }}=0$. The most resilient and distant systems such as HD 95872 show no difference when replaced with the 2:1 equivalent.

\subsubsection{Planet masses and multiplicities}

We now consider two complications to our treatment of the planet masses in our simulations. First, we have set the planet masses in the simulations to equal $M \sin I$ of the observed planet, which on average will underestimate the planet mass by a factor of 1.6 , and is a strict lower limit on the mass. To explore the effect of the true mass being higher, we have re-run the single-planet simulations (to calculate $f_{\text {hab,1P }}$ ) for HD 95872 and HD 72659 with planetary masses increased by factors of 1.4, 1.7 and 2.0. For HD 95872 there is no difference in the outcome. For HD 72659, where the giant's pericentre is closer to the $\mathrm{HZ}$, we find a small reduction in $f_{\text {hab }, 1 \mathrm{P}}$ of $0.02-0.06$. A larger mass is marginally more disruptive if the pericentre is within a few Hill radii of the HZ, but because the Hill radius is a weak function of mass (Equation 3 ) the effect is not very strong.

When considering $f_{\text {rhab }}$, we expect that the peak of the eccentricity distribution after scattering will shift to slightly higher values with increasing planet-star mass ratio. However, we also expect that the mass ratio between planets in the system is more significant in setting the final eccentricity distribution and the history of scattering. We note here that soon Gaia will provide a measure of the true mass of many Jupiter analogues (Sozzetti et al. 2008; Perryman et al. 2014; Ranalli et al. 2018), permitting a refinement to the estimation of $f_{\text {hab,1P }}$ and $f_{\text {rhab }}$ by repeating these simulations with the real planetary masses.

The second complication is that we have reduced the set of planetary mass ratios considered to two choices: strictly equal masses (3E), or a hierarchical Solar System analogue (4H). Although we have shown that this simplification can reproduce the observed eccentricity distribution of the giant exoplanets, in reality mass ratios will fall on a continuum; this greatly increases the parameter space to investigate. Here, we simply note the following: With our dichotomous distribution of mass ratios, for a given eccentricity the distribution of $f_{\text {rhab }}$ is bimodal (figure 8), having a high-survivability peak from the $4 \mathrm{H}$ systems and a lowsurvivability peak from the $3 \mathrm{E}$ systems. We would then expect, for example, a 2:1:1 mass ratio to be intermediate between the $4 \mathrm{H}$ and the $3 \mathrm{E}$ in destructibility, and with a continuum of mass ratios, we expect the $f_{\text {rhab }}$ distribution to be less bimodal.

Finally, the number of planets in our simulations was fixed at either three or four. Adding more planets will likely be more destructive to bodies in the $\mathrm{HZ}$ if the planets are comparable in mass to the largest planet, since there will be more possibilities of scattering a large planet onto an orbit with a small pericentre, and also more secular resonances to destabilise the $\mathrm{HZ}$ at a distance. However, if the additional planets are smaller (neptune-mass), there should be a weaker effect, as we have shown that the dominant 


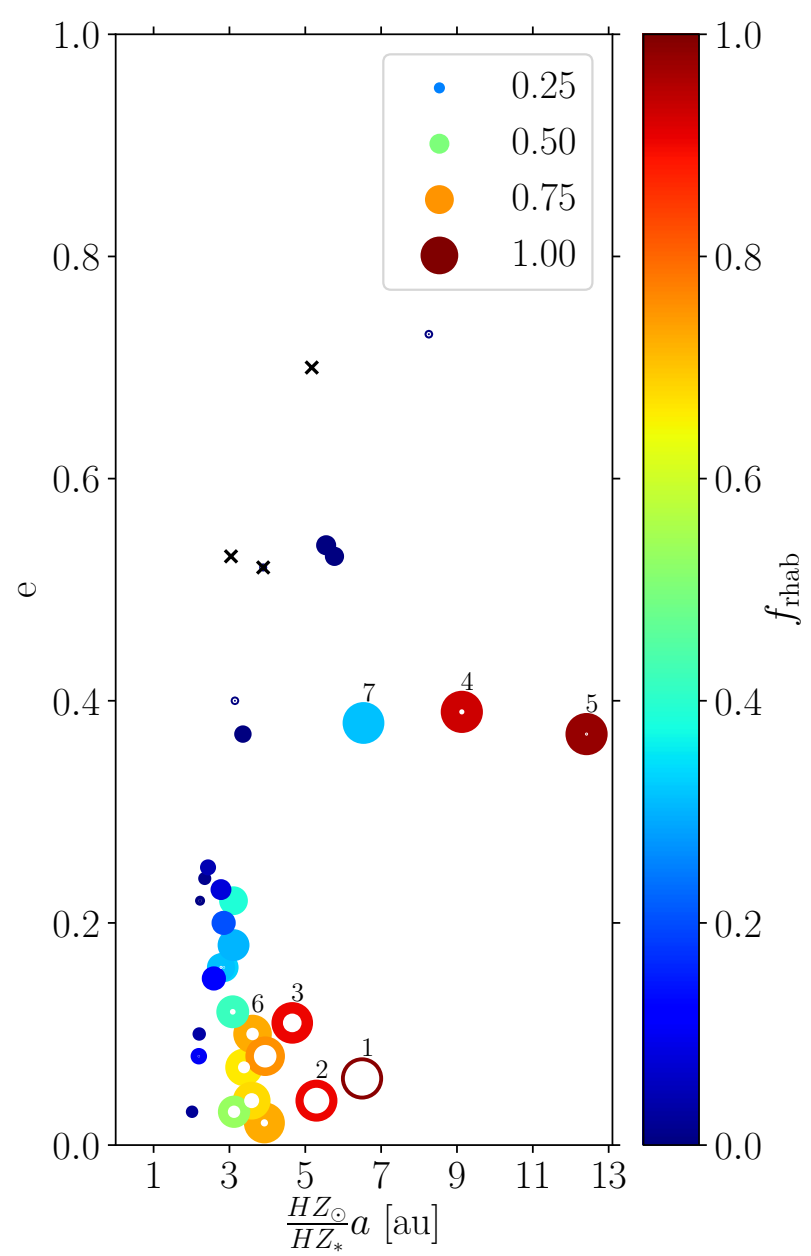

Figure 14. The figure shows the resilient habitability, and its uncertainty, of all the simulated systems. The systems are marked with their planets' semimajor axes rescaled so that the midpoint of the systems' HZ corresponds to the midpoint of the HZ in the Solar System, and with their reported eccentricities. The colour of each symbol is set by the median of $f_{\text {rhab- }}$ distribution. The inner and outer radii of annular symbols scale linearly with the $\pm 1 \sigma$ values of $f_{\mathrm{rhab}}$-distribution. The crosses indicate systems where the $1 \sigma$ upper limit is less than 0.05 . Thus, the blue crosses are very bad, small blue dots are bad, the narrow brown/red annuli are good and the wide annuli give a range of possible outcomes. We have have highlighted the best potential systems for follow-up observations; they are as follows: 1) HD 95872 2) HD 154345 3) HD 102843 4) HD 25015 5) GJ 328 6) HD 6718 7) HD 150706.

mechanism for destabilising the $\mathrm{HZ}$ is not the entry of neptunemass planet into it (Section 6.1).

\section{FUTURE OBSERVATIONS}

Finally, we can make some comments as to which of the systems we have studied provide the best prospects for the detection of planets in the HZ, based on their resilient habitability. All the loweccentricity systems in Group 1 make good candidates for observations as they all have high median $f_{\text {rhab-values. Particularly good }}$ are the ones where the lower end of the orange interval in Figure 7 is high: HD 95872, HD 154345 and HD 102843. These are highlighted in Figure 14, where we show the resilient habitability for all of the systems we have studied as a function of the giant planet's eccentricity and distance to the HZ. This figure should be compared to figure 12 of Carrera et al. (2016), which shows the median resilient habitability for a generic system. The broad features are the same - high resilient habitability for low eccentricity and wide orbit planets - but we wish to emphasize two points: the large range in resilient habitability arising from different runs in the same system; and the fact that different systems close to each other in parameter space on this Figure can have different values of resilient habitability, in part because we have included the mass of the known planet as a third parameter.

We also highlight in Figure 14 some other systems that offer good prospects for follow up. HD 6718 and HD 150706 have large uncertainties on their eccentricities, and if the true eccentricities are at the lower end of the allowed ranges then their resilient habitability is high (see Figure 9). HD 25015 and GJ 328 are of high eccentricity $(e \sim 0.4)$ but distant from the HZ. They show strongly bimodal distributions of $f_{\text {rhab }}$ : if their past history involved ejection of an equal-mass planet then $f_{\text {rhab }} \approx 0$, but if they have ejected a lower-mass planet then their resilient habitability is high. The $f_{\text {rhab }}$ distribtions of these seven systems of interest are shown in Figure 15.

In the introduction we made a point about utilizing Gaia to constrain the orbital plane of the giant planets and using that to identify edge-on systems that will also show a strong RV signal from any potential Earth-like planet. The systems we have studied, typically at distances of a few $10 \mathrm{~s}$ of pc, will have a good enough signal-to-noise to detect the astrometric reflex motion of the star from the known Jovian planets (Ranalli et al. 2018, figure 2). Furthermore, the orbital inclination of the giant planet in these systems will be constrained to $\sigma_{\cos I} \sim 0.1$ (Ranalli et al. 2018, figure 9 and table 5).

Finally, we consider how the orbit of the stars through the Galaxy may affect the habitability of the systems. Kokaia \& Davies (2019) showed how the passage of a star and its planetary system through giant molecular clouds - which may trigger mass extictions - depends on the star's orbit in the Galaxy. We obtain the positions and velocities of the 7 highlighted systems from Gaia DR2 (Gaia Collaboration et al. 2018) and integrate them to determine their Galactic orbits (see Kokaia \& Davies 2019 for details). We find that for all of the systems, their orbits in the Galaxy lead to excursions of more than $100 \mathrm{pc}$ from the Galactic plane. Comparing to (Kokaia \& Davies 2019) we find that all of them have encounters with giant molecular clouds at a similar rate to the Sun: between once every $500 \mathrm{Myr}$ and $3 \mathrm{Gyr}$.

\section{SUMMARY}

We have selected 34 systems containing a single massive planet orbiting an FGK-dwarf beyond the habitable zone (HZ). We simulated the dynamical history of these systems considering initial multiple-planet systems comprising either three equal-mass planets (3E) or four planets having a mass hierarchy of the four gas giants of our Solar System $(4 \mathrm{H})$. These systems pass through a phase of instability where planets are ejected via planet-planet scattering leaving other planets on more bound and more eccentric orbits. One can probe the entire observed eccentricity range with $3 \mathrm{E}$ and $4 \mathrm{H}$ systems. Low-eccentricity planets are produced mostly by $4 \mathrm{H}$ systems, whereas all systems containing a planet with an eccentricity, $e>0.5$ are derived exclusively from $3 \mathrm{E}$ systems.

We select the subset of the runs which give eccentricities of the 


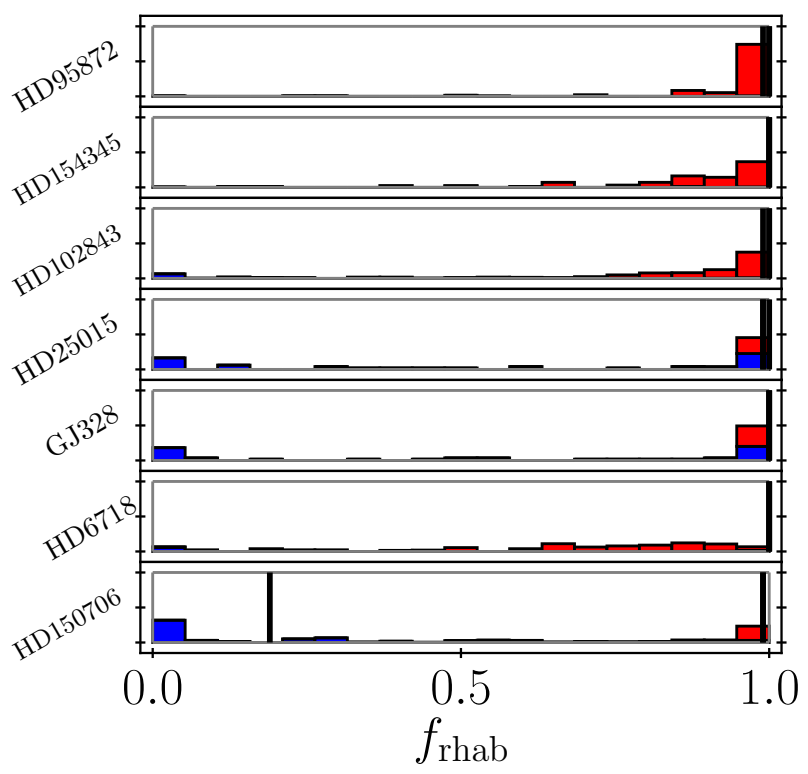

Figure 15. The binned, resampled data used to produce the $f_{\text {rhab }}$ distributions in figure 7. The histograms are normalized such that for each system the blue and the red bins together add up to one. The blue bins show the number of $3 \mathrm{E}$ runs and the red bins show the $4 \mathrm{H}$ runs. The black lines show the bounds of the $f_{\text {hab, } 1 \mathrm{P} \text {-values. }}$

inner planet matching observations. Our simulations include test particles in the habitable zone, to represent terrestrial planets. We measure the fraction of test particles located within the $\mathrm{HZ}$ which survive the dynamical evolution, and term this fraction the resilient habitability, $f_{\text {rhab }}$. We also compute the fraction of test particles within the $\mathrm{HZ}$ which would be removed from the HZ by the single planet as observed today, and refer to this as the present-day habitability, $f_{\text {hab, } 1 \mathrm{P}}$.

For the $3 \mathrm{E}$ runs, the resulting instability tends to be much more violent resulting in higher eccentricities and planets often entering the $\mathrm{HZ}$ and clearing it out. For $4 \mathrm{H}$, on the other hand, the two most massive planets rarely enter the $\mathrm{HZ}$ with the most massive one only doing so in systems where the observed planet is very close to the $\mathrm{HZ}$ boundary. This leaves the HZ mostly intact as the lower mass planets cannot directly eject the test particles. The systems, shown in figure 3, are placed into one of three groups:

Group 1: Systems where the planet has a much larger semimajor axis than the outer edge of the $\mathrm{HZ}$ and/or has a low eccentricity. These systems all have high values of $f_{\text {hab,1P }}$ and the median values of the $f_{\text {rhab }}$ distribution also tend to be high. However, some of the systems in the group have distributions of $f_{\text {rhab }}$ that go down to much lower values as can be seen in figure 7 . In all cases, $f_{\text {rhab }}$

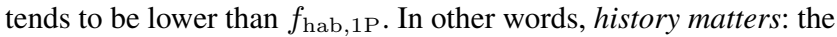
dynamical past is more damaging than present perturbations of the observed planet.

Group 2: Systems where the planet has a slightly larger semimajor axis than the outer edge of the $\mathrm{HZ}$ and/or has a high eccentricity which brings it very close to the HZ. These planets are much more damaging to objects within the HZ. Systems in Group 2 generally show much lower values of $f_{\text {hab,1P }}$ than seen in Group 1 . Values of $f_{\text {rhab }}$ are even lower owing to the extra damaging effects of the earlier phase of planet-planet scattering.

Group 3: Systems that could fit in either Group 1 or Group 2, due to the planets having large uncertainties in their eccentricities which leads to a large range of possible $f_{\text {hab,1P }}$ and $f_{\text {rhab }}$ values. For these systems we recalculate the $f_{\text {rhab }}$ distribution by considering three narrower ranges of eccentricities centred around the mean and $+/-1 \sigma$ values of the observed eccentricities as shown in figure 9 . We see two systems that show very large differences between the distribution of $f_{\text {rhab }}$ if the eccentricity is high, and that if it is low; these are HD 6718 and HD 150706, and therefore better determination of these eccentricities is needed.

Even in the $4 \mathrm{H}$ runs where the most-massive planet does not enter the $\mathrm{HZ}$, the planet can do significant damage to the $\mathrm{HZ}$ when its semi-major axis changes during the scattering phase, as then mean-motion resonances can sweep over the $\mathrm{HZ}$ and pump up the eccentricity of the test particles until they have a close encounter with the gas giant and get ejected or until the test particle hits the star. This is consistent with models for the depletion of the asteroid belt in the Solar System (Izidoro et al. 2016; Clement et al. 2019).

In contrast to the mean-motion resonances, the secular resonances very rarely do any damage to the habitable zone. This is because for most systems, having the secular resonances inside the habitable zone requires the two planets to be 4-7 mutual hill radii apart. Whilst this is a stable configuration for circular coplanar orbits, it is rarely stable after an instability. The planets can in principle end up at the right semi-major axes but they will have eccentric orbits which generally leads to either one of them eventually getting ejected or a reconfiguration such that the secular resonances move away from the HZ.

We provide a list of systems containing at least one known giant planets most likely to be able to host an Earth-like planet: HD 95872, HD 154345, HD 102843, HD 25015, GJ 328, HD 6718 and HD 150706. HD 95872 is the clearly best candidate as can be seen in figure 14.

\section{ACKNOWLEDGMENTS}

The authors are supported by the project grant 2014.0017 "IMPACT" from the Knut and Alice Wallenberg Foundation. The simulations were performed on resources provided by the Swedish National Infrastructure for Computing (SNIC) at Lunarc, which we can contribute thanks to grants from The Royal Physiographic Society of Lund. We thank Ross P. Church for comments on the manuscript. We also thank the referee for their feedback which helped improve the paper.

\section{REFERENCES}

Abramov O., Mojzsis S. J., 2009, Nature, 459, 419

Agnew M. T., Maddison S. T., Thilliez E., Horner J., 2017, MNRAS, 471, 4494

Agnew M. T., Maddison S. T., Horner J., 2018, MNRAS, 477, 3646

Anglada-Escudé G., López-Morales M., Chambers J. E., 2010, ApJ, 709, 168

Antoniadou K. I., Voyatzis G., 2016, MNRAS, 461, 3822

Bashi D., Helled R., Zucker S., Mordasini C., 2017, A\&A, 604, A83

Batygin K., Laughlin G., 2008, ApJ, 683, 1207

Batygin K., Laughlin G., 2015, Proceedings of the National Academy of Science, 112,4214

Benedict G. F., et al., 2006, AJ, 132, 2206

Bitsch B., Crida A., Libert A. S., Lega E., 2013, A\&A, 555, A124

Bitsch B., Izidoro A., Johansen A., Raymond S. N., Morbidelli A., Lambrechts M., Jacobson S. A., 2019, A\&A, 623, A88 
Boisse I., et al., 2012, A\&A, 545, A55

Bolmont E., Libert A.-S., Leconte J., Selsis F., 2016, A\&A, 591, A106

Bryan M. L., Knutson H. A., Lee E. J., Fulton B. J., Batygin K., Ngo H., Meshkat T., 2019, AJ, 157, 52

Buchhave L. A., Bitsch B., Johansen A., Latham D. W., Bizzarro M., Bieryla A., Kipping D. M., 2018, ApJ, 856, 37

Carrera D., Davies M. B., Johansen A., 2016, MNRAS, 463, 3226

Carter-Bond J. C., O'Brien D. P., Raymond S. N., 2014, in Haghighipour N., ed., IAU Symposium Vol. 293, Formation, Detection, and Characterization of Extrasolar Habitable Planets. pp 229-234, doi:10.1017/S174392131301288X

Chambers J. E., 1999, MNRAS, 304, 793

Chambers J. E., 2001, Icarus, 152, 205

Chambers J. E., Wetherill G. W., Boss A. P., 1996, Icarus, 119, 261

Childs A. C., Quintana E., Barclay T., Steffen J. H., 2019, MNRAS, 485, 541

Chyba C. F., 1990, Nature, 343, 129

Clement M. S., Raymond S. N., Kaib N. A., 2019, The Astronomical Journal, 157, 38

Davies M. B., Adams F. C., Armitage P., Chambers J., Ford E., Morbidelli A., Raymond S. N., Veras D., 2014, in Beuther H., Klessen R. S., Dullemond C. P., Henning T., eds, Protostars and Planets VI. p. 787 (arXiv:1311.6816), doi:10.2458/azu_uapress_9780816531240ch034

Denham P., Naoz S., Hoang B.-M., Stephan A. P., Farr W. M., 2019, MNRAS, 482, 4146

Dressing C. D., Spiegel D. S., Scharf C. A., Menou K., Raymond S. N., 2010, ApJ, 721, 1295

Endl M., et al., 2016, ApJ, 818, 34

Feng F., et al., 2019, The Astrophysical Journal Supplement Series, 242, 25

Ford E. B., Rasio F. A., 2008, ApJ, 686, 621

Gaia Collaboration et al., 2018, Astronomy and Astrophysics, 616, A1

Georgakarakos N., Eggl S., Dobbs-Dixon I., 2018, ApJ, 856, 155

Gilmozzi R., Spyromilio J., 2007, The Messenger, 127, 11

Giuppone C. A., Morais M. H. M., Correia A. C. M., 2013, MNRAS, 436, 3547

Gladman B., 1993, Icarus, 106, 247

Grazier K. R., 2016, Astrobiology, 16, 23

Hadden S., Lithwick Y., 2018, AJ, 156, 95

Halliday A. N., 2008, Philosophical Transactions of the Royal Society of London Series A, 366, 4163

Hansen B. M. S., 2009, ApJ, 703, 1131

Hansen B. M. S., 2017, MNRAS, 467, 1531

Hara N. C., Boué G., Laskar J., Delisle J. B., Unger N., 2019, MNRAS, 489, 738

Horner J., Jones B. W., 2008, International Journal of Astrobiology, 7, 251

Horner J., Jones B. W., 2009, International Journal of Astrobiology, 8, 75

Horner J., Jones B. W., Chambers J., 2010, International Journal of Astrobiology, 9, 1

Innanen K. A., Zheng J. Q., Mikkola S., Valtonen M. J., 1997, AJ, 113, 1915

Izidoro A., Raymond S. N., Pierens A., Morbidelli A., Winter O. C., Nesvorny‘ D., 2016, The Astrophysical Journal, 833, 40

Jacobsen S. B., 2005, Annual Review of Earth and Planetary Sciences, 33, 531

Jenkins J. S., et al., 2017, MNRAS, 466, 443

Johansen A., Davies M. B., Church R. P., Holmelin V., 2012, ApJ, 758, 39

Jones B. W., Sleep P. N., Chambers J. E., 2001, A\&A, 366, 254

Jurić M., Tremaine S., 2008, ApJ, 686, 603

Kaib N. A., Raymond S. N., Duncan M. J., 2011, ApJ, 742, L24

Kane S. R., Torres S. M., 2017, AJ, 154, 204

Kokaia G., Davies M. B., 2019, Monthly Notices of the Royal Astronomical Society, p. 945

Kopparapu R. K., Ramirez R. M., SchottelKotte J., Kasting J. F., DomagalGoldman S., Eymet V., 2014, ApJ, 787, L29

Kürster M., Trifonov T., Reffert S., Kostogryz N. M., Rodler F., 2015, A\&A, 577, A103

Laakso T., Rantala J., Kaasalainen M., 2006, A\&A, 456, 373
Lambrechts M., Morbidelli A., Jacobson S. A., Johansen A., Bitsch B., Izidoro A., Raymond S. N., 2019, A\&A, 627, A83

Laskar J., Gastineau M., 2009, Nature, 459, 817

Leite A. C. O., Martins C. J. A. P., Molaro P., Monai S., Alves C. S., Silva T. A., the ESPRESSO Science Team 2018, arXiv e-prints, p. arXiv: 1812.06796

Linsenmeier M., Pascale S., Lucarini V., 2015, Planet. Space Sci., 105, 43

Lissauer J. J., et al., 2011, ApJS, 197, 8

Lucy L. B., Sweeney M. A., 1971, AJ, 76, 544

Machida M. N., Kokubo E., Inutsuka S.-I., Matsumoto T., 2010, MNRAS, 405,1227

Malmberg D., Davies M. B., Heggie D. C., 2011, MNRAS, 411, 859

Mardling R. A., Aarseth S. J., 2001, MNRAS, 321, 398

Marmier M., et al., 2013, A\&A, 551, A90

Matsumura S., Ida S., Nagasawa M., 2013, ApJ, 767, 129

Menou K., Tabachnik S., 2003, ApJ, 583, 473

Ment K., Fischer D. A., Bakos G., Howard A. W., Isaacson H., 2018, AJ, 156,213

Morbidelli A., Chambers J., Lunine J. I., Petit J. M., Robert F., Valsecchi G. B., Cyr K. E., 2000, Meteoritics and Planetary Science, 35, 1309

Mordasini C., Alibert Y., Benz W., 2009, A\&A, 501, 1139

Moutou C., et al., 2011, A\&A, 527, A63

Mullally F., et al., 2015, ApJS, 217, 31

Murray C. D., Dermott S. F., 1999, Solar system dynamics

Mustill A. J., Wyatt M. C., 2012, MNRAS, 419, 3074

Mustill A. J., Davies M. B., Johansen A., 2017, MNRAS, 468, 3000

Mustill A. J., Davies M. B., Johansen A., 2018, MNRAS, 478, 2896

O'Brien D. P., Morbidelli A., Levison H. F., 2006, Icarus, 184, 39

O'Brien D. P., Izidoro A., Jacobson S. A., Raymond S. N., Rubie D. C., 2018, Space Science Reviews, 214, 47

Pepe F. A., et al., 2010, in Ground-based and Airborne Instrumentation for Astronomy III. p. 77350F, doi:10.1117/12.857122

Perryman M., Hartman J., Bakos G. Á., Lindegren L., 2014, ApJ, 797, 14

Petrovich C., 2015, ApJ, 808, 120

Piso A.-M. A., Youdin A. N., 2014, ApJ, 786, 21

Piso A.-M. A., Youdin A. N., Murray-Clay R. A., 2015, ApJ, 800, 82

Ragusa E., Rosotti G., Teyssandier J., Booth R., Clarke C. J., Lodato G., 2018, MNRAS, 474, 4460

Ranalli P., Hobbs D., Lindegren L., 2018, A\&A, 614, A30

Rauer H., et al., 2014, Experimental Astronomy, 38, 249

Raymond S. N., Izidoro A., 2017, Icarus, 297, 134

Raymond S. N., Mandell A. M., Sigurdsson S., 2006, Science, 313, 1413

Raymond S. N., O'Brien D. P., Morbidelli A., Kaib N. A., 2009, Icarus, 203, 644

Rey J., et al., 2017, A\&A, 601, A9

Rickman E. L., et al., 2019, arXiv e-prints,

Robertson P., Endl M., Cochran W. D., MacQueen P. J., Boss A. P., 2013, ApJ, 774, 147

Rowan D., et al., 2016, ApJ, 817, 104

Shen Y., Turner E. L., 2008, ApJ, 685, 553

Shikita B., Koyama H., Yamada S., 2010, ApJ, 712, 819

Skidmore W., TMT International Science Development Teams Science Advisory Committee T., 2015, Research in Astronomy and Astrophysics, 15,1945

Sozzetti A., Casertano S., Lattanzi M. G., Spagna A., Morbidelli R., Pannunzio R., Pourbaix D., Queloz D., 2008, in Jin W. J., Platais I., Perryman M. A. C., eds, IAU Symposium Vol. 248, A Giant Step: from Millito Micro-arcsecond Astrometry. pp 256-259 (arXiv: 0711.4903), doi:10.1017/S1743921308019200

Spiegel D. S., Raymond S. N., Dressing C. D., Scharf C. A., Mitchell J. L., 2010, ApJ, 721, 1308

Stassun K. G., Collins K. A., Gaudi B. S., 2017, AJ, 153, 136

Thommes E. W., Matsumura S., Rasio F. A., 2008, Science, 321, 814

Veras D., Armitage P. J., 2005, ApJ, 620, L111

Ward P. D., Brownlee D., Krauss L., 2000, Physics Today, 53, 62

Wittenmyer R. A., et al., 2014, ApJ, 783, 103

Wittenmyer R. A., Bergmann C., Horner J., Clark J., Kane S. R., 2019, MNRAS, 484, 4230 
18 G. Kokaia, M. B. Davies, A. J. Mustill

Zakamska N. L., Pan M., Ford E. B., 2011, MNRAS, 410, 1895

Zeebe R. E., 2015, ApJ, 798, 8

Zhu W., Wu Y., 2018, AJ, 156, 92 